\title{
The exocyst complex and intracellular vesicles mediate soluble protein trafficking to the primary cilium
} \\ Niewiadomski $\mathrm{P}^{1, *}$ \\ ${ }^{1}$ Centre of New Technologies, University of Warsaw, Warsaw, Poland \\ ${ }^{2}$ Faculty of Biology, University of Warsaw, Warsaw, Poland \\ ${ }^{3}$ Department of Medicine, Stanford University School of Medicine, Stanford, CA, USA \\ ${ }^{4}$ Current address: IGM Biosciences, Inc., Mountain View, CA, USA \\ ${ }^{5}$ Department of Biochemistry, Stanford University School of Medicine, Stanford, CA, USA \\ ${ }^{*}$ Correspondence: p.niewiadomski@cent.uw.edu.pl
}

\section{Abstract}

Efficient transport of proteins into the primary cilium is a crucial step for many signaling pathways. Dysfunction of this process can lead to the disruption of signaling cascades or cilium assembly, resulting in developmental disorders and cancer. Previous studies on ciliary trafficking were mostly focused on the membrane-embedded receptors. In contrast, how soluble proteins are delivered into the cilium is poorly understood. In our work, we identify the exocyst complex as a key player in the ciliary trafficking of soluble Gli transcription factors. Considering that the exocyst mediates intracellular vesicle transport, we demonstrate that soluble proteins, including Gli2/3 and Lkb1, can use the endosome recycling machinery for their delivery to the primary cilium. Finally, we identify GTPases: Rab14, Rab18, Rab23, and Arf4 involved in vesicle-mediated Gli protein ciliary trafficking. Our data pave the way for a better understanding of ciliary transport and uncover novel transport mechanisms inside the cell.

\section{Introduction}

Hedgehog $(\mathrm{Hh})$ signaling is essential for embryonic patterning and organ morphogenesis ${ }^{1}$. Malfunctions of this pathway can lead to developmental disorders and cancer. The expression of Hh target genes is controlled by Gli transcription factors: Gli1 which acts as an activator, and Gli2/Gli3, which display both activator and repressor functions ${ }^{2}$.

Processing of Gli transcription factors to activator and repressor forms requires their efficient transport to the primary cilium, which integrates proteins necessary to a variety of Gli modifications $^{3-8}$. Cilia are indispensable for the transduction of the $\mathrm{Hh}$ signal and the translocation of Gli activators into the nucleus ${ }^{9}$. In humans, defects in the ciliary function and the trafficking of ciliary proteins often result in developmental defects associated with the dysfunction of the Hh/Gli cascade.

Gli proteins are large and slowly diffusing proteins, so it is puzzling how they accumulate at the cilium within a mere few minutes upon signal reception ${ }^{10}$. This accumulation is a result of a three-step process: (1) targeted transport to the cilium base, (2) gated entry through a diffusion barrier, and (3) active trafficking along the cilium. The mechanisms of Gli transition across the diffusion barrier and the model of transport from the base to the tip are relatively 
well-described ${ }^{11-13}$. However, it is still unclear how Gli proteins are delivered so quickly and precisely from the cytoplasm to the cilium base.

42 Most previous studies on protein delivery to the ciliary base were focused on membrane proteins. Three different transport routes have been described for their delivery from the Golgi complex to the primary cilium ${ }^{14}$. Some ciliary proteins first reach the plasma membrane and then move to the ciliary membrane by lateral transport ${ }^{15}$. Others reach the base of the cilium using regulated vesicular transport, either directly or through the recycling trafficking pathway ${ }^{16}$.

The process of protein trafficking to the primary cilium is supported by many players involved in endocytosis and the vesicle transport machinery ${ }^{17,18}$. Prominent among them are small GTPases, which act as molecular switches that allow for the guidance of their associated vesicles ${ }^{19-21}$. In addition to GTPases, the protein ciliary trafficking depends on several multiprotein complexes, such as the BBsome ${ }^{22,23}$ and the exocyst ${ }^{24,25}$. The exocyst is a conserved protein complex that mediates the tethering of secretory vesicles to the plasma membrane ${ }^{26}$. It interacts with the ciliary transport machinery to transport transmembrane proteins necessary for ciliogenesis and signaling ${ }^{16,27,28}$.

In our quest to identify the molecular machinery that delivers Gli proteins to the cilium base, we performed a proteomic analysis of Gli3 interactors. Interestingly, among Gli3-binding proteins, we detected several exocyst subunits ${ }^{26}$. Loss-of-function assays show the dependence of Gli2 and Gli3 ciliary localization on the exocyst. Consistent with the role of this complex in vesicle trafficking, we show that Gli2 uses intracellular vesicles as trafficking vehicles. In addition, several small GTPases, including Rab14, Rab18, Rab23, and Arf4, regulate the ciliary transport of Gli2. Finally, we show that this vesicle-based transport machinery is used for the ciliary delivery of Lkb1, another soluble protein that concentrates at cilia. 


\section{Results}

\section{The exocyst complex interacts with Gli3}

To identify proteins that help guide Gli proteins to the primary cilium, we immunoprecipitated proteins that interact with Gli3 in cells treated with the Smoothened (Smo) agonist SAG ${ }^{29}$. Cells were separated into "nuclear" and "cytoplasmic" fractions and then immunoprecipitated with anti-Gli3 antibodies. The eluates were separated using SDS-PAGE, and prominent bands were submitted for MS-based protein identification (Fig. 1A).

We identified 473 high confidence Gli3 interactors by rejecting frequent IP/MS contaminants based on the CRAPome database ${ }^{30}$. In this dataset, we found well-known Gli interaction partners, such as SuFu, Kif7, and Xpo $7^{31-34}$. The dataset was enriched for proteins involved in intraciliary and vesicle transport, chromatin remodeling, and DNA repair (Fig. 1B) and contained components of multi-subunit ciliary transport complexes, including the BBSome and the exocyst (Fig. 1C, Supplementary Table S1).

Because exocyst, a multi-subunit protein complex involved in vesicle transport and docking ${ }^{35}$, had previously been implicated in the trafficking of proteins to primary cilia, we decided to focus on its components as potential mediators of the Gli proteins delivery to the cilium base. The exocyst has mostly been studied in the context of its binding to intracellular vesicles and the plasma membrane, but the subunits that were specifically enriched in the Gli3 interactome are positioned away from the putative lipid-binding surface of the complex, consistent with Gli3 being a soluble, rather than a lipid-embedded protein (Fig. 1D).

In agreement with the proteomic data, Gli3, as well as Gli2, co-immunoprecipitate with Sec5 (Fig. 2A). Moreover, Sec5 and Gli2 tightly colocalize in cells, as shown using the proximity ligation assay (Fig. 2B). Similarly, overexpressed Sec3, Sec5, and Sec8 interact with the constitutively active Gli2 mutants Gli2(P1-6A) (Fig. 2C, D) ${ }^{36}$. We decided to use Gli2(P1-6A) in most experiments because it localizes to cilia in the absence of upstream activation, allowing us to study its trafficking independently of the transport of membrane proteins regulating endogenous Gli proteins, such as Smo and Ptch ${ }^{37,38}$.

To identify the Gli2 domain responsible for interaction with the exocyst, we performed coimmunoprecipitation of Sec3/5/8 with the N-terminal domain of Gli2 and a construct lacking the $\mathrm{N}$-terminus. The exocyst subunits interact with the N-terminus of Gli2 (HA-Gli2-N) but interact only weakly with Gli2(P1-6A)- $\Delta N$ (Fig. 2E, F).

\section{Trafficking of Gli2 to cilia depends on the exocyst}

Because the exocyst is required for the trafficking of some ciliary proteins, we hypothesized that the loss-of-function of the exocyst could impair Gli ciliary localization. To test this assertion, we knocked down individual exocyst subunits in cells expressing Gli2(P1-6A). Both shRNA- (Fig. 3A) and siRNA-mediated knockdown (Fig. 3B) of exocyst subunits resulted in a significant reduction of Gli2(P1-6A) ciliary localization (Fig. 3C and D).

Similarly, mislocalization of Sec5 using the mitochondrial trap ${ }^{39}$ impairs the ciliary trafficking of Gli2(P1-6A). We fused Sec5 with the mitochondrial protein Tom20 and mScarlet and coexpressed the resulting Tom20-mScarlet-Sec5 construct with Gli2(P1-6A) (Fig. 4A). We 
observed a reduced Gli2 ciliary level in cells overexpressing the Tom20-mScarlet-Sec5 mitochondrial trap, compared to those overexpressing two negative control constructs Tom20-mScarlet and mScarlet-Sec5 (Fig. 4B).

Finally, the exocyst inhibitor endosidin2 reduces Gli2(P1-6A) ciliary localization in the stable cell line after just two hours of treatment (Fig. 4C).

Because the exocyst binds to Gli2 mostly via its N-terminal domain (Fig 2E, F), we suspected that removing the $\mathrm{N}$-terminus would impair Gli2 ciliary accumulation. Accordingly, we observed a strong reduction of the Gli2(P1-6A)- $\Delta \mathrm{N}$ mutant localization in the primary cilium compared to the full-length protein (Fig. 4D).

Having demonstrated that the exocyst is required for the trafficking of Gli to cilia, we wondered if the localization of the exocyst is affected by Hh pathway activation. Indeed, the treatment with SAG increases the amount of Sec3 and Sec5 at the ciliary base suggesting that the exocyst is co-transported with Gli proteins upon pathway activation (Fig. 4E).

\section{Gli2 associates with intracellular vesicles}

While the best-known role of the exocyst complex is the transport of vesicle-embedded membrane proteins, our results suggest that soluble cytoplasmic Gli proteins may also use the exocyst as a vehicle for intracellular trafficking. We, therefore, wondered if Gli proteins use vesicles for their transport into the cilium. To verify this hypothesis, we used super-resolution AiryScan microscopy to image cells co-expressing HA-Gli2(P1-6A) and EGFP-Sec5, and surprisingly, we observed Gli2 around Sec5-positive vesicle-like structures. It suggests that Gli2 could accumulate on the surface of vesicles, where it could interact directly with the exocyst (Fig. 5A).

We also looked at Gli2 localization by immunogold electron microscopy. In HEK293T cells overexpressing EGFP-Gli2(P1-6A), we observed EGFP-positive clusters adjacent to membrane vesicle-like structures (Fig. 5B).

To check if Gli-positive structures represent intracellular vesicles, we isolated vesicles using cell fractionation. HA-Gli2(P1-6A), endogenous Gli3, and Sec5 co-fractionated with the endosome marker EEA1 in the endosomal fraction. ERK was used as the cytoplasmic control marker. The total abundance of proteins in fractions we showed by silver staining (Fig. 5C).

The most likely explanation for our results is that Gli proteins are transported on the surface of vesicles towards the ciliary base. The two potential sources of these vesicles are the Golgi apparatus via the exocytic pathway ${ }^{40,41}$ and the plasma membrane by endocytosis ${ }^{42-44}$. Firstly, we inhibited endocytosis using two inhibitors: dynasore ${ }^{45}$ and pitstop $2^{46}$ in cells expressing constitutively active $\mathrm{Gli} 2$. Surprisingly, after $2 \mathrm{~h}$ of dynasore treatment, we observed an almost complete inhibition of Gli2 ciliary accumulation. This effect was independent of Smo because treatment with two Smo inhibitors cyclopamine and vismodegib did not affect the Gli2(P1-6A) ciliary level (Fig. 5E and Fig. S2).

If the dynasore effects are a consequence of the reduced rate of new vesicle formation, we would expect these effects to be fully reversible once the proper formation of vesicles is 
restored. We used a pulse-chase assay with $2 \mathrm{~h}$ vismodegib + dynasore treatment, and then we washed out dynasore from the media and collected cells at several time points. We observed a clear recovery of Gli2 ciliary transport within $1 \mathrm{~h}$ from the dynasore washout (Fig. 5D).

We also used another inhibitor of endocytosis - pitstop2. Because of its lethal effect on NIH3T3 fibroblasts in less than 30min, we treated cells with pitstop2 for $15 \mathrm{~min}$, followed by a $30 \mathrm{~min}$ incubation without the drug. Similar to the dynasore effects, we observed a decrease of Gli2 ciliary level in pitstop2-treated cells (Fig. 5F).

To determine if the vesicle transport from the cis-Golgi was also important for Gli2 ciliary trafficking, we treated stable HA-Gli2(P1-6A) cells with brefeldin A, a Golgi-disrupting drug ${ }^{47}$. We did not observe changes in Gli2 ciliary localization after $2 \mathrm{~h}$ treatment (Fig. 5G).

The stimulation of target gene transcription by Gli2 is enhanced by its localization at the cilium $^{9,48}$. We expected that dynasore would inhibit $\mathrm{Hh}$ target gene transcription in cells stably expressing the Gli2(P1-6A). Indeed, the expression of the Hh target gene Gli1 was decreased after dynasore treatment, although the expression of HA-Gli2(P1-6A) was unchanged (Fig. $5 \mathrm{H}$ ).

\section{Rab and Arf proteins mediate Gli2 transport}

The trafficking of vesicles in cells is guided by the reversible association of small GTPases, especially from the Rab and Arf families ${ }^{18,49}$. Because their association with vesicles and associated proteins is transient, we hypothesized that under the stringent conditions of our initial co-IP/MS, the Gli-associating GTPases may have been washed away from the bait protein. Thus, we performed another co-IP/MS, with less stringent detergents, using HAGli2(P1-6A) as bait in cells that either had normal cilia or were devoid of cilia by means of overexpression of a dominant-negative mutant Kif3a motor ${ }^{50}$. We expected the GTPases promoting Gli ciliary trafficking to be associated with Gli2 in ciliated, but not in unciliated cells (Fig. 6A).

We identified 200 high-confidence interactors (<10\% FDR in the CRAPome database) including the same well-known regulators of Gli, such as SuFu, Kif7, Xpo7, and Spop 34,32,51,52, as well as component proteins of the cilium and basal body (Fig. 6B, Supplementary Table S2). Among proteins associated with Gli2(P1-6A) in ciliated cells were Rab14, Rab5c, Rab11b, Rab18, and Arf4 (Fig. 6C). In addition, we tested two other Rab-family GTPases: the well-known Hh regulator Rab23 $3^{53-55}$ and Rab8, which cooperates with the exocyst ${ }^{56,57}$.

Initially, we established by co-IP that Rab14, Rab18, Rab23, and Arf4 proteins interact with Gli2(P1-6A) (Fig. 7A). In contrast, two Rab GTPases that had been implicated in ciliary trafficking of membrane proteins: Rab8 and Rab11a, do not strongly bind to Gli2(P1-6A) (Fig. S3A).

Subsequently, we performed loss-of-function experiments using shRNA and CRISPR/Cas9 mutagenesis. The knockdown of Rab14, Rab18, and Arf4 caused the reduction of the Gli2(P16A) ciliary level (Fig. 7B-D). Likewise, the CRISPR/Cas9-mediated Rab14, Rab18, and Rab23 knockout also significantly decreased the Gli2(P1-6A) ciliary accumulation (Fig. 7E). Moreover, we engineered cell lines expressing dominant-negative Rab23S51N and Arf4T31N mutants 
184 from doxycycline-inducible promoters. Consistent with shRNA- and CRISPR/Cas9-based 185 experiments, we observed a significant decrease of Gli2(P1-6A) ciliary accumulation in cells 186 expressing Arf4 and Rab23 mutants(Fig.7F).

\section{The trafficking of Lkb1, but not Ubxn10, depends on endocytosis and the exocyst}

188 To establish if the mechanism of transport to cilia by endocytic vesicles is unique to Gli 189 proteins or more common, we imaged several HA or GFP tagged soluble ciliary candidate 190 proteins: HA-Dvl2 ${ }^{58}$, Kap3a-EGFP ${ }^{59}$, HA-Lkb160, HA-Mek161, HA-Nbr1 ${ }^{62}$, HA-Raptor ${ }^{63}$, Tbx3191 GFP 64 , and Ubxn10-GFP65. Only two proteins clearly localized at cilia in NIH/3T3: Ubxn10-GFP, 192 and HA-Lkb1 (Fig. 8A and Fig. S4).

193 To examine if the ciliary serine-threonine kinase Lkb1 uses an analogous transport mechanism, 194 we treated stable expressing HA-Lkb1 cells with dynasore and observed decreased Lkb1 ciliary 195 level (Fig. 8D). Similar to Gli2, ciliary accumulation of HA-Lkb1 also dropped after the shRNA 196 knockdown of Sec3/5/8 (Fig. 8B). Accordingly, we detected HA-Lkb1 in the endosomal fraction 197 (Fig. 7G). Finally, we observed using co-IP that Lkb1 binds to the exocyst subunits (Fig. 8F).

198 Another soluble ciliary protein that we studied was Ubxn10. Dynasore treatment did not 199 negatively affect the ciliary trafficking of Ubxn10-GFP (Fig. 8E). Unlike for Gli2(P1-6A), we 200 observed no effect of Sec5 knockdown on Ubxn10 ciliary localization (Fig. 8C). Consistent with 201 these results, we detected Ubxn10 predominantly in the cytosolic cell fraction (Fig. 8H). 


\section{Discussion}

204 The cilium is an essential organelle that relays environmental signals to the nucleus.

205 Nevertheless, the mechanism of the signaling protein delivery to cilia is still poorly 206 understood, especially for soluble proteins. To gain a better understanding of cytoplasmic 207 proteins' transport to cilia we studied Gli transcription factors, large soluble proteins that 208 accumulate at the tip of the cilium before their conversion into transcriptional activators $5,9,11$.

209 Using proteomic screening, we found that Gli proteins interact with the exocyst, a complex 210 implicated in ciliary delivery of membrane receptors ${ }^{16,35}$. We found that loss-of-function of 211 the exocyst by RNAi, mitochondrial trap, or drug treatment decreases ciliary localization of 212 the constitutively active mutant Gli2(P1-6A) independently of their effect on transmembrane 213 Hh signaling proteins Ptch and Smo.

214 On a molecular level, we show that the $\mathrm{N}$-terminal region of Gli proteins binds to the 215 subcomplex I of the exocyst ${ }^{26,66}$. This agrees with our data and published reports suggesting 216 agree that the $\mathrm{N}$-terminal domain is necessary for the Gli proteins ciliary accumulation ${ }^{5,9,11}$. 217 The N-terminus is, however, not sufficient for Gli ciliary transport, with other domains, 218 particularly the central domain of $\mathrm{Gli} 2 / 3^{5,9}$ likely participating in other stages of ciliary 219 translocation.

220 Our results suggest that soluble cytoplasmic proteins, like Gli2/3, can use the exocyst as a 221 vehicle for intracellular trafficking. The exocyst was shown to collaborate with the BLOC-1 222 complex and IFT20 in the transport of membrane proteins polycystin-2 and fibrocystin to 223 cilia ${ }^{16}$. However, IFT20 does not interact with HA-Gli2(P1-6A) (Fig. S3A). This suggests that the 224 exocyst may mediate Gli protein ciliary trafficking independently of IFT20, which implies that 225 the pathways directing membrane and soluble cilium components are somewhat divergent. 226 Importantly, the exocyst can be transported to the cilium despite IFT20 loss-of-function ${ }^{16}$.

227 Consistent with the requirement of the exocyst in the transport of Gli2 to cilia, it appears that $228 \mathrm{Gli2}$ is associated, at least transiently, with intracellular vesicles. Interestingly, the subunits of 229 the exocyst that most strongly interact with Gli2 are positioned away from the putative lipid230 facing surface of the complex ${ }^{26,66}$, indicating that the exocyst may form a tether between 231 vesicle lipids and soluble proteins. Structural ciliary proteins had been previously found to be 232 attached to the outer surfaces of intracellular vesicles carrying ciliary membrane proteins in 233 Chlamydomonas ${ }^{67}$. We now provide functional data that corroborate and extend these 234 findings. Protein delivery by vesicles to the cilium is persistent and essential for maintaining 235 proper cilium function and structure ${ }^{68,69}$. Thus, the strategy of using vesicles as universal 236 carriers of proteins, both soluble and membrane-embedded, to cilia, solves the logistical 237 problem of homing many protein classes onto the tiny cilium base.

238 The trafficking of vesicles in cells is coordinated by the small GTPases from the Rab and Arf 239 families. Intriguingly, we found that Rab14, Rab18, Rab23, and Arf4, interact with Gli2 and are 240 essential for its accumulation in the ciliary compartment. The Rab14 GTPase localizes at early 241 endosomes and plays a role in protein exchange between the endosomes and the Golgi 242 compartment ${ }^{70-73}$, and exocytic vesicle targeting ${ }^{74}$. On the other hand, Rab18 is usually 243 associated with the endoplasmic reticulum and lipid droplets ${ }^{75,76}$. Intriguingly, we identify 
COPI and TRAPP complex components in Gli2(P1-6A) and Gli3 interactomes, and these complexes have been implicated in lipid droplet recruitment of Rab18 ${ }^{77}$. This suggests that Gli may recruit Rab18 via TRAPPII and COPI to promote ciliary trafficking. Interestingly, all three of the above GTPases: Rab18, Rab14, and Arf4, were recently identified as proximity interactors of the cilium base-localized kinase Ttbk2 ${ }^{78}$, strengthening the case for their involvement in the targeting of Gli-laden vesicles to the cilium.

Finally, Rab23 had previously been implicated in Hh signaling and ciliary transport of receptors ${ }^{79}$. Rab23 is described as a negative regulator of the Hh pathway but several different mechanisms have been proposed, from affecting Smo to directly regulating Gli proteins ${ }^{54,80,53}$. Here, we propose Rab23 as one of the key players in the trafficking of Gli transcription factors into the primary cilium. This is consistent with the recently discovered role of Rab23 in the transport of another soluble protein, Kif17, to primary cilia and with the ciliary and early endosome enrichment of Rab23 ${ }^{81,82}$.

In addition to Rab family GTPases, we found Gli2 to associate with Arf4, which functions in sorting ciliary cargo at the Golgi and is a crucial regulator of ciliary receptor trafficking 83,84 . Arf4 binds the ciliary targeting signal of rhodopsin and controls the assembly of the Rab11aRabin8-Rab8 module for the proper delivery of cargo to the ciliary base ${ }^{85}$. Although Rab8 and Rab11a were found to cooperate with both the exocyst and Arf4 ${ }^{85}$ in the targeting of ciliary cargos, we found that the expression of dominant-negative Rab8 and Rab11a did not negatively affect Gli2 ciliary accumulation, with Rab8 DN actually promoting higher Gli2 accumulation in cilia (Fig. S3B). Moreover, we did not find Rab8 or Rab11a among interactors of Gli2 and Gli3. Instead, among Gli2 interactors was a Rab11a ortholog Rab11b, which had also been implicated in ciliogenesis and found to associate with Rab8 ${ }^{86,87}$. Disentangling the roles of the two Rab11 orthologs as well as Rab8/Rabin8 in the trafficking of soluble ciliary components will be an interesting subject for future studies.

Many of the implicated Rab/Arf proteins had been known to associate both with Golgi-derived exocytic vesicles and with plasma membrane-derived endosomes. To decipher the relative importance of these two potential vesicle sources, we used pharmacological inhibitors to show that Gli2 is likely delivered to cilia via endocytic vesicle trafficking rather than the canonical secretory pathway.

274 In addition to Gli2, other soluble ciliary proteins can adopt a similar transport mechanism. 275 Specifically, we show that Lkb1 levels at primary cilia drop upon exocyst loss-of-function and inhibition of endocytosis. Like Gli2, Lkb1 associates with intracellular vesicles and interacts with the exocyst. In contrast, another soluble ciliary component Ubxn10 localizes at the cilium normally in cells depleted of Sec5 or treated with dynasore. This suggests that while the vesicle-mediated transport is important for the ciliary localization of some cytoplasmic proteins, others use different routes of ciliary trafficking.

281 In summary, we describe a novel mechanism for the transport of soluble cytoplasmic proteins 282 to primary cilia, which relies on the association of these proteins with dynamically cycling endocytic vesicles. While we identify several key players in the ciliary trafficking of these 
285 process. In particular, it will be interesting to discover potential similarities and differences

286 between the canonical ciliary targeting pathways for membrane proteins, such as polycystin

287 2, fibrocystin, Smo, and rhodopsin with those described here for soluble ciliary proteins. Our 288 work brings us closer to gaining a broad understanding of ciliary trafficking and the 289 coordinated transport of proteins among membrane compartments.

\section{Acknowledgments}

291 We would like to thank Marta Miączyńska, Małgorzata Maksymowicz, Jarosław Cendrowski, 292 and the members of the CeNT Bio PI discussion group, and the Laboratory of Molecular and 293 Cellular Signalling for insightful discussion and helpful suggestions. We thank M. Raman, A. 294 Moon, and P. Avasthi for sharing their reagents with us and Addgene contributors for making 295 their plasmids available (see Table 1). This work was supported by the following grants from 296 the National Science Centre (NCN): SONATA BIS 2014/14/E/NZ3/00033 and PRELUDIUM 297 2018/29/N/NZ3/01523. 


\section{Materials and methods}

300

301

302

303

304

305

306

307

308

309

310

311

312

313

314

315

316

317

\section{Constructs and molecular cloning}

Gli2/3 constructs were cloned based on the Gli2(P1-6A) mutant previously described ${ }^{36}$ tagged with the $\mathrm{N}$-terminal 3xHA. Initially, Gli2 fragments were amplified by PCR and then cloned into the pENTR2B (Life Technologies) vector by Gibson assembly ${ }^{88}$ using the NEBuilder ${ }^{\oplus}$ HiFi DNA Assembly Master Mix (NEB). Subsequently, the constructs were shuttled into pEF/FRT/V5DEST (Life Technologies) using the Gateway method (Gateway LR Clonase II mix; Life Technologies). Plasmids with Sec3/5/8, Rab8/11/14/18, and Arf4 on the pEGFP vector were ordered from the Addgene site (Tab. 1). Rab23 wild type and mutant cDNA sequences were obtained by DNA synthesis (DNA Strings; Thermo) and cloned by Gibson assembly into the LT3GEPIR plasmid ordered from addgene (Tab. 1). Tom20 sequence was amplified from mouse cDNA and then fused with mScarlet cloned from pmScaret (addgene, Tab. 1) and Sec5 by Gibson assembly in the pEGFP-C3 vector with the EGFP sequence removed by restriction digestion. Other soluble proteins sequences of Dvl2, Nbr1, Mek1, Lkb1, Raptor were amplified from mouse cDNA and cloned into the pENTR2B with 3XHA tag vector by Gibson assembly. Ubnx10 was cloned from pHAGE-NGFP-UBXD3 - gift from M. Raman ${ }^{65}$. Tbx3 was cloned from the construct with Tbx3-Myc - a gift from A. Moon ${ }^{64}$. pEGFP-Kap3a was a gift from P. Avasthi and pEGFP-Rab11a was a gift from M. Miaczynska.

Tab. 1. Plasmids used in our experiments ordered from the addgene site

\begin{tabular}{|lllll|}
\hline $\mathbf{1}$ & Plasmid name & Addgene No. & Gift from & References \\
\hline $\mathbf{2}$ & pEGFP-C3-Sec5 & $\# 53755$ & Channing Der & 89 \\
\hline $\mathbf{3}$ & pEGFP-C3-Sec8 & $\# 53756$ & Channing Der & 89 \\
\hline $\mathbf{4}$ & pmScarlet_C1 & $\# 53758$ & Channing Der & 89 \\
\hline $\mathbf{5}$ & pLKO.1-TRC cloning vector & $\# 10878$ & David Root & 91 \\
\hline $\mathbf{6}$ & pLKO.1- blast & $\# 25566$ & Keith Mostov & 92 \\
\hline $\mathbf{7}$ & lentiCas9-Blast & $\# 52962$ & Feng Zhang & 93 \\
\hline $\mathbf{8}$ & LT3GEPIR & $\# 111177$ & Johannes Zuber & 94 \\
\hline $\mathbf{9}$ & lentiGuide-Puro & $\# 52963$ & Feng Zhang & 93 \\
\hline $\mathbf{1 0}$ & pRSV-Rev & $\# 12253$ & Didier Trono & 95 \\
\hline $\mathbf{1 1}$ & pMDLg/pRRE & $\# 12251$ & Didier Trono & 95 \\
\hline $\mathbf{1 2}$ & pMD2.G & $\# 12259$ & Didier Trono & unpublished \\
\hline $\mathbf{1 3}$ & EGFP-Rab14 & $\# 49549$ & Marci Scidmore & 96 \\
\hline $\mathbf{1 4}$ & EGFP-Rab18 & $\# 49550$ & Marci Scidmore & 96 \\
\hline $\mathbf{1 5}$ & Arf4-GFP & $\# 39556$ & Paul Melancon & 97 \\
\hline $\mathbf{1 6}$ & pCAG/hArf4(T31N)-HA & $\# 79405$ & Kazuhisa Nakayama & 98 \\
\hline $\mathbf{1 7}$ & GFP-rab11 DN & $\# 12678$ & Richard Pagano & 99 \\
\hline $\mathbf{1 8}$ & pGFP-Rab8A & $\# 24898$ & Maxence Nachury & 100 \\
\hline $\mathbf{1 9}$ & pGFP-Rab8A[T22N] & $\# 24899$ & Maxence Nachury & 100 \\
\hline $\mathbf{2 0}$ & pLenti-IFT20-EGFP & $\# 118032$ & Ken Ichi Takemaru & unpublished \\
\hline
\end{tabular}




\section{Cell culture}

HEK293T (ATCC) and NIH/3T3 Flp-In (Thermo) cells were maintained in media composed of DMEM (high glucose; Biowest), sodium pyruvate (Thermo), stable glutamine (Biowest), nonessential amino acids (Thermo), 10\% fetal bovine serum (EurX), and penicillin/streptomycin solution (Thermo). HA-Gli2(P1-6A) and HA-LKB1 NIH3T3 stable cell lines were generated using the Flp-In system according to the manufacturer's protocols (Thermo Fisher). Stable cell lines were reselected with hygromycin on every other passage to preserve selection pressure.

To stimulate ciliogenesis the cells were cultured in the same medium but containing $0.5 \%$ FBS for $24 \mathrm{~h}$ before fixing. For activation of the Hh pathway, we used SAG (Smoothened agonist) treatment $200 \mathrm{nM}$ for $24 \mathrm{~h}$. Transient transfections of cells we performed using the JetPrime reagent (Polyplus) according to the manufacturer's protocol.

All inhibitors were suspended in DMSO and used with indicated times. The following concentrations of inhibitors were used: dynasore ( $40 \mu \mathrm{M}$, Sigma), endosidin2 (200 $\mu \mathrm{M}$, Sigma), pitstop2 (30 $\mu \mathrm{M}$, Sigma), brefeldin A $(5 \mu \mathrm{g} / \mathrm{ml}$, Sigma).

\section{Large scale co-IP/MS on Gli3}

$\mathrm{NIH} / 3 \mathrm{T3}$ cells were cultured to confluence on $5015 \mathrm{~cm}$ dishes and starved overnight to promote ciliogenesis. They were treated with 100nM SAG for $4 \mathrm{~h}$. The cells were fractionated into nuclear and cytoplasmic fractions as previously described ${ }^{101}$. Each fraction was immunoprecipitated overnight with $150 \mu \mathrm{L}$ Dynabeads-Protein G (Invitrogen) covalently crosslinked with goat-anti-Gli3 (AF3690; R\&D Systems; 30 $\mu$ g antibody per fraction). The beads were washed with the following buffers: harsh RIPA lysis buffer $(50 \mathrm{mM}$ Tris $\mathrm{pH} 7.4,150 \mathrm{mM} \mathrm{NaCl}$, $2 \%$ Nonidet P-40, 500mM LiCl, 1mM DTT, 0.25\% sodium deoxycholate, $0.1 \%$ SDS, protease and phosphatase inhibitors), RIPA lysis buffer supplemented with $0.8 \mathrm{M}$ urea, and mild $0.1 \%$ NP-40 lysis buffer (50mM Tris pH 7.4, 150mM NaCl, 0.1\% Nonidet P-40, 1mM DTT, 1\% glycerol, phosphatase inhibitors). The samples were eluted from beads using preheated $2 x$ Laemmli sample buffer without DTT at $85^{\circ} \mathrm{C}$ for $5 \mathrm{~min}$. The samples were then reduced and alkylated using DTT and iodoacetamide and loaded onto a 6\% SDS-PAGE gel. The gel was stained using the GelCode Blue reagent (Pierce) and prominent bands were excised using a sterile scalpel and submitted for further processing to MS Bioworks (Ann Arbor, MI). The bands were destained and subjected to in-gel digest using trypsin. Each gel digest was analyzed by nano LC/MS/MS with a Waters NanoAcquity HPLC system interfaced to a ThermoFisher LTQ Orbitrap Velos. Peptides were loaded on a trapping column and eluted over a $75 \mu \mathrm{m}$ analytical column at $350 \mathrm{~nL} / \mathrm{min}$; both columns were packed with Jupiter Proteo resin (Phenomenex). The mass spectrometer was operated in data-dependent mode, with MS performed in the Orbitrap at 60,000 FWHM resolution and MS/MS performed in the LTQ. The fifteen most abundant ions were selected for MS/MS. Data were searched using a local copy of Mascot with the following parameters: Enzyme: Trypsin, Database: IPI Mouse v3.75 (forward and reverse appended with common contaminants), Fixed modification: Carbamidomethyl (C), Variable modifications: Oxidation (M), Acetyl (N-term, K), Pyro-Glu (N-term Q), Deamidation $(N, Q)$, Phospho $(S, T, Y)$, GlyGly $(K)$, Mass values: Monoisotopic, Peptide Mass Tolerance: 10 ppm, Fragment Mass Tolerance: 0.5 Da, Max Missed Cleavages: 2. Mascot DAT files were parsed into the Scaffold algorithm for validation, filtering, and to create a nonredundant list 
per sample. Data were filtered using a minimum protein value of $90 \%$, a minimum peptide value of $50 \%$ (Prophet scores), and requiring at least two unique peptides per protein.

To determine high-confidence Gli3 interactors, we rejected all proteins found in more than $10 \%$ of negative control affinity purification/MS experiments in the CRAPome database ${ }^{102}$ (FDR < 10\%). Enrichment of proteins representing specific Gene Ontology terms was performed using PANTHER with GO-Slim Cellular Component and GO-Slim Biological Process terms ${ }^{103}$.

\section{Large scale co-IP/MS on HA-Gli2 (P1-6A) in ciliated and non-ciliated cells}

$\mathrm{NIH} / 3 \mathrm{~T} 3$ cells stably expressing HA-Gli2 (P1-6A) were transduced either with the control vector or with a retroviral vector encoding the dominant-negative variant of Kif3a (headless - amino acids 441-701 of the mouse Kif3a; dnKif3a) and selected with puromycin to eliminate untransduced cells. Each cell line was expanded from a single clone and ciliogenesis or lack thereof was verified by immunofluorescence.

Both cell lines were starved for $36 \mathrm{~h}$ and lysed in a gentle lysis buffer (50mM Tris pH 7.4, $150 \mathrm{mM} \mathrm{NaCl}, 0.1 \%$ Nonidet P-40, 5\% glycerol, protease and phosphatase inhibitors) and scraped at $4^{\circ} \mathrm{C}$. The lysate was clarified for $30 \mathrm{~min}$ at $15,000 \mathrm{xg}$ and the supernatant was immunoprecipitated for $2 \mathrm{~h}$ at $4^{\circ} \mathrm{C}$ with Dynabeads-protein $\mathrm{G}$ covalently coupled to the rat anti-HA antibody (Roche). The beads were washed $3 \times 5 \mathrm{~min}$. with the lysis buffer and $1 \times 5 \mathrm{~min}$ with the lysis buffer with the addition of $350 \mathrm{mM} \mathrm{NaCl}$ (total $\mathrm{NaCl}$ concentration $500 \mathrm{mM}$ ). Protein was eluted from beads using $2 x$ Laemmli sample buffer at $37^{\circ} \mathrm{C}$ for $30 \mathrm{~min}$ with vigorous mixing (500rpm).

Eluted proteins were submitted for mass spectrometric protein identification to MS Bioworks (Ann Arbor, MI). The entire amount of sample was separated $\sim 1.5 \mathrm{~cm}$ on a $10 \%$ Bis-Tris Novex mini-gel (Invitrogen) using the MES buffer system. The gels were stained with coomassie and excised into ten equally sized segments. Gel segments were processed using a robot (ProGest, DigiLab) with the following protocol:?Washed with $25 \mathrm{mM}$ ammonium bicarbonate followed by acetonitrile. Reduced with $10 \mathrm{mM}$ dithiothreitol at $60^{\circ} \mathrm{C}$ followed by alkylation with $50 \mathrm{mM}$ iodoacetamide at RT. Digested with trypsin (Promega) at $37^{\circ} \mathrm{C}$ for $4 \mathrm{~h}$. Quenched with formic acid and the supernatant was analyzed directly without further processing.

The gel digests were analyzed by nano LC/MS/MS with a Waters M-class HPLC system interfaced to a ThermoFisher Fusion Lumos. Peptides were loaded on a trapping column and eluted over a $75 \mu \mathrm{m}$ analytical column at $350 \mathrm{~nL} / \mathrm{min}$; both columns were packed with Luna $\mathrm{C} 18$ resin (Phenomenex). A 30min gradient was employed (5h LC/MS/MS per sample). The mass spectrometer was operated in data-dependent mode, with MS and MS/MS performed in the Orbitrap at 60,000 FWHM resolution and 15,000 FWHM resolution, respectively. APD was turned on. The instrument was run with a 3s cycle for MS and MS/MS. Data were searched using a local copy of Mascot with the following parameters: Enzyme: Trypsin, Database: Swissprot Mouse (concatenated forward and reverse plus common contaminants), Fixed modification: Carbamidomethyl (C), Variable modifications: Oxidation (M), Acetyl (Protein Nterm), Deamidation (NQ), Mass values: Monoisotopic, Peptide Mass Tolerance: 10 ppm, Fragment Mass Tolerance: $0.02 \mathrm{Da}$, Max Missed Cleavages: 2. Mascot DAT files were parsed 
401 into the Scaffold software for validation, filtering, and to create a nonredundant list per 402 sample. Data were filtered at $1 \%$ protein and peptide level FDR and requiring at least two 403 unique peptides per protein.

\section{Viral transduction}

405 For lentivirus production, we transfected HEK293T cells with pRSV-rev, pMDLg/pRRE, pMD2.G 406 lentiviral packaging vectors (addgene, Tab. 1) and the construct encoding our protein or 407 shRNA or sgRNA of interest, and then after 2 days, we collected the virus-containing medium 408 and added it to target cells. We used puromycin to select transduced cells.

\section{9 siRNA mediated knockdown}

410 For siRNA-mediated knockdown of Sec5, we used the Sec5 ON-TARGET plus siRNA set of four 411 siRNAs with non-targeting controls (Horizon Dharmacon). For siRNA transfection, we used 412 Lipofectamine RNAiMAX (Thermofisher). Each siRNA was introduced at $40 \mathrm{pmol} /$ well on a 24413 well plate for $48 \mathrm{~h}$.

414 shRNA mediated knockdown

415 shRNAs were cloned into pLKO.1-TRC cloning vector (Tab. 1). Targeting sequences were 416 designed using the BlockIT software from the Thermo-Fisher website.

\section{CRISPR-Cas9-mediated mutagenesis}

418 CRISPR-Cas9-mediated mutagenesis was performed on NIH/3T3 Flp-In cells stably expressing 419 HA-Gli2(P1-6A) and Cas9 (Tab. 1). sgRNA sequences were designed using the Broad Institute 420 sgRNA designer tool ${ }^{104}$ and cloned into the pLentiGuide-puro vector (addgene, Tab. 1). We 421 transduced the target cells with lentiviruses carrying the sgRNA of interest and either fixed $42272 \mathrm{~h}$ later or subjected to antibiotic selection.

423 Tab. 2. Sequences used for shRNA knockdown, CRISPR edition, and qPCR primers.

\begin{tabular}{|ll|}
\hline name & sequence \\
\hline shRNA-Sec3 & GGAGGTGGACCAGATTGAACT \\
\hline shRNA-Sec5 & GCAGGAGCTAAAGCAGATTGT \\
\hline shRNA-Sec8 & CGGTTACACGGAGCTACTATA \\
\hline shRNA-Rab18 & TATCATGGCAGTGAGTATTTG \\
\hline shRNA-Arf4 & CGGTTACACGGAGCTACTATA \\
\hline shRNA-Luciferase & GCTGGAGAGCAACTGCATAAG \\
\hline sgRNA Rab23 & AAAGACTACAAGAAAACCAT \\
\hline sgRNA Rab14 & CATATAACCACTTAAGCAGC \\
\hline sgRNA Rab18 & ATACTCATCATCGGCGAGAG \\
\hline sgRNA Arf4 & GATCGTGAAAGAATCCAGGA \\
\hline qPCR Rab14 F & GGTTCAGAGCGGTTACACG \\
\hline qPCR Rab14 R & TGAGATTCCTTGCGTCTGTC \\
\hline qPCR Rab18 F & GCACGCAAGCATTCTATGTTG \\
\hline qPCR Rab18 R & AGCTTGACTCCTTTGTTCTGG \\
\hline
\end{tabular}




\begin{tabular}{|ll|}
\hline qPCR Arf4 $\mathbf{F}$ & AGGATCTGCCAAACGCTATG \\
\hline qPCR Arf4 $\mathbf{R}$ & CCTCATACAGACCAGTTCCTTG \\
\hline qPCR Sec3 F & TCGCGCTGAGAAAAGATGAC \\
\hline qPCR Sec3 $\mathbf{R}$ & TTCTTGCCAGCTTTGCAGAC \\
\hline qPCR Sec5 F & CGGAGGTGCAAGTTTTCA AG \\
\hline qPCR Sec5 $\mathbf{R}$ & GCATGGAGGTCGGAAAGA TAC \\
\hline qPCR Sec8 $\mathbf{F}$ & AATTGACCACAGCCATTCGC \\
\hline qPCR Sec8 $\mathbf{R}$ & TCATCCCGTTTGCAATGCAG \\
\hline
\end{tabular}

Immunostaining and microscopy.

426 Cells were cultured on glass coverslips. After low-serum starvation to promote ciliogenesis, we fixed cells in $4 \%[\mathrm{w} / \mathrm{v}]$ paraformaldehyde in PBS for $15 \mathrm{~min}$ at room temperature (RT) and then washed $3 \times 10 \mathrm{~min}$ in phosphate buffer saline (PBS). Subsequently, cells were blocked and permeabilized in $5 \%[\mathrm{w} / \mathrm{v}]$ donkey serum in $0.2 \%[\mathrm{w} / \mathrm{v}]$ Triton X-100 in PBS. We incubated cells with the primary antibodies diluted in blocking buffer overnight at $4^{\circ} \mathrm{C}$. Next, we washed the coverslips $3 \times 10 \mathrm{~min}$ with $0.05 \%[\mathrm{w} / \mathrm{v}$ ] Triton X-100 in PBS, followed by incubation with secondary antibodies in the blocking buffer for 1 hour at RT. Cells were washed as above and mounted onto slides using a fluorescent mounting medium with DAPI (ProLong Diamond, Thermo). We acquired images on an inverted Olympus IX-73 fluorescent microscope equipped with a 63x UPLANAPO oil objective and the Photometrics Evolve 512 Delta camera. For superresolution microscopy, we used the Zeiss LSM800 confocal microscope with the Airyscan detector and Plan Apochromat 63x/1.4 Oil DIC objective.

For the quantitative analysis of fluorescence intensities, images were acquired with the same settings of exposure time, gain, offset, and illumination. Fluorescent intensities were measured in a semi-supervised manner by a custom ImageJ script. To calculate the Gli ciliary accumulation, we calculated the $\log _{10}$ values of the ratios of intensities of the fluorescent signal at the tip of the primary cilium and the surrounding background in each cell.

Tab. 3. Antibodies used for western blot, immunofluorescence staining, and proximity ligation assay

\begin{tabular}{|llll|}
\hline Antibody & Application & Ref No. \\
\hline Primary antibodies & Western blot (WB), Immunofluorescence (IF) & \\
\hline anti-HA High Affinity & WB (1:1000); IF (1:2000) & Roche & 11867423001 \\
\hline anti-HA & WB (1:1000) & BioLegend & 901501 \\
\hline anti-GFP & WB (1:1000) & Genetex & GTX113617 \\
\hline anti-Arl13b & IF (1:2000) & Proteintech & $17711-1-A P$ \\
\hline anti-Sec5 & WB (1:500); IF (1:200); & Proteintech & $12751-1-A P$ \\
\hline anti-Sec5 & PLA & & \\
\hline anti-Sec3 & WB (1:2000) & Proteintech & 66011-1-Ig \\
\hline anti-Sec8 & WB (1:1000); IF (1:500) & Proteintech & $11690-1-A P$ \\
\hline
\end{tabular}




\begin{tabular}{|c|c|c|c|}
\hline anti- $\alpha$-tubulin & WB (1:1000) & Sigma & T6199 \\
\hline anti-beta-actin & WB (1:1000) & Sigma & A1978-100UL \\
\hline anti-Gli2 & WB (1:1000) & \multicolumn{2}{|c|}{ home-made by Davids biotechnologie } \\
\hline anti-Gli2 & PLA & R\&D Systems & AF3635 \\
\hline anti-Gli3 & WB (1:1000) & R\&D Systems & AF3690 \\
\hline anti-Pericentrin & IF $(1: 200)$ & BD Biosciences & 611814 \\
\hline anti-EEA1 & WB (1:1000) & BD Biosciences & 610456 \\
\hline anti-acetylated tubulin & IF $(1: 1000)$ & Sigma & T6793 \\
\hline \multicolumn{4}{|l|}{ Secondary antibodies } \\
\hline anti-mouse alexa-488 & IF $(1: 1000)$ & Jackson Immunoresearch & $715-545-151$ \\
\hline anti-rabbit alexa-488 & IF $(1: 1000)$ & Jackson Immunoresearch & $711-545-152$ \\
\hline anti-rat alexa-488 & IF $(1: 1000)$ & Jackson Immunoresearch & $712-545-153$ \\
\hline anti-rabbit alexa-Cy3 & IF $(1: 1000)$ & Jackson Immunoresearch & $711-165-152$ \\
\hline anti-mouse alexa-594 & IF $(1: 1000)$ & Jackson Immunoresearch & $715-585-151$ \\
\hline anti-rabbit alexa-594 & IF $(1: 1000)$ & Jackson Immunoresearch & $711-585-152$ \\
\hline anti-rat alexa-647 & IF $(1: 1000)$ & Jackson Immunoresearch & $712-605-153$ \\
\hline HRP anti-mouse & WB $(1: 2500)$ & BioLegend & 405306 \\
\hline HRP anti-rabbit & WB $(1: 2500)$ & BioLegend & 406401 \\
\hline HRP anti-goat & WB (1:2500) & Sigma & A5420 \\
\hline
\end{tabular}

447 We performed co-immunoprecipitation using Pierce Anti-HA Magnetic Beads (Life 448 Technologies) or using Dynabeads-protein $G$ (Thermo) magnetic beads with primary 449 antibodies (anti-GFP Genetex No\#GTX113717; anti-Sec5 Proteintech No\#12751-1-AP) cross450 linked using dimethyl pimelimidate (Life Technologies).

451 For the production of whole-cell lysates, cells were lysed in $4^{\circ} \mathrm{C}$ in lysis buffer $(50 \mathrm{mM}$ Tris at $452 \mathrm{pH} 7.4,1 \% \mathrm{NP}-40[\mathrm{v} / \mathrm{v}], 150 \mathrm{mM} \mathrm{NaCl}, 0.25 \%$ sodium deoxycholate $[\mathrm{v} / \mathrm{v}]$, protease inhibitor 453 cocktail [1× EDTA-free protease inhibitors, Sigma], 10mM NaF). 1/10 part of the clarified lysate 454 was saved as an input fraction, and the rest was subjected to immunoprecipitation.

455 After adding beads, binding of the protein of interest was performed overnight with gentle 456 rotation at $4^{\circ} \mathrm{C}$. The next day, beads were washed $4 \times 10 \mathrm{~min}$ in $4^{\circ} \mathrm{C}$ in the same lysis buffer to 457 remove unbound proteins, and complexes were eluted off the beads using $2 x$ SDS sample 458 buffer at $37 \mathrm{C}$ for $30 \mathrm{~min}$. We analyzed the composition of eluent using the SDS-PAGE and 459 Western Blot method.

461 Proteins were denaturated for $30 \mathrm{~min}$ at $65^{\circ} \mathrm{C}$ and resolved by SDS-PAGE. Afterward, we 462 performed electrotransfer onto a nitrocellulose membrane. Immunocomplexes were 463 detected using an enhanced chemiluminescence detection system (Clarity or Clarity Max, Bio$464 \mathrm{rad}$ ) on Amersham Imager 680 and 800 as 16-bit grayscale TIFF files. The molecular weight of 465 proteins was estimated with pre-stained protein markers (Bio-rad). 


\section{Proximity Ligation Assay}

467 We performed the proximity ligation assay ${ }^{105}$ using the Duolink PLA Kit (Merck) according to 468 the manufacturer's protocol. Anti-Sec5 and anti-Gli2 primary antibodies (Tab. 3) were used to 469 detect sites of interaction between the proteins in NIH/3T3 Flp-In cells.

470 Endosome Isolation

471 The Trident Endosome Isolation Kit (Genetex) was used to fractionate cell lysates according to 472 the manufacturer's protocol.

\section{Electron Microscopy}

474 HEK293 cells expressing EGFP-Gli2(P-16A) were fixed on the dish with 4\% PFA in $0.2 \mathrm{M}$ 475 phosphate buffer and $0.25 \%$ sucrose. The samples were sent to Biocenter Oulu Electron 476 Microscopy Core Facility and there processed for EM and immunogold labeled with anti-GFP. 477 Imaging was performed on Sigma HD VP FE-SEM equipped with ET-SE and In-lens SE detectors, 478 VPSE G3 detector for low vacuum mode, and 5Q-BSD detector.

\section{Data analysis}

480 The statistical data analysis was performed using Microsoft Excel and R/RStudio. For the 481 processing of the fluorescence images, we used the FiJi/ImageJ suite. Statistical significance 482 was calculated using Student's t-test for experiments involving two experimental groups, or 483 ANOVA and Tukey posthoc test for multiple comparisons. 


\section{References}

1. Briscoe, J. \& Thérond, P.P. The mechanisms of Hedgehog signalling and its roles in development and disease. Nat. Rev. Mol. Cell Biol. 14, 416-429 (2013).

2. Niewiadomski, P. et al. Gli Proteins: Regulation in Development and Cancer. Cells 8, (2019).

3. Huangfu, D. et al. Hedgehog signalling in the mouse requires intraflagellar transport proteins. Nature 426, 83-87 (2003).

4. Goetz, S. C. \& Anderson, K. V. The primary cilium: a signalling centre during vertebrate development. Nat. Rev. Genet. 11, 331-344 (2010).

5. Zeng, H., Jia, J. \& Liu, A. Coordinated translocation of mammalian Gli proteins and suppressor of fused to the primary cilium. PloS One 5, e15900 (2010).

6. Tukachinsky, H., Lopez, L. V. \& Salic, A. A mechanism for vertebrate Hedgehog signaling: recruitment to cilia and dissociation of SuFu-Gli protein complexes. J. Cell Biol. 191, 415-428 (2010).

7. Niewiadomski, P., Zhujiang, A., Youssef, M. \& Waschek, J. A. Interaction of PACAP with Sonic hedgehog reveals complex regulation of the hedgehog pathway by PKA. Cell. Signal. 25, 2222-2230 (2013).

8. Barzi, M., Berenguer, J., Menendez, A., Alvarez-Rodriguez, R. \& Pons, S. Sonichedgehog-mediated proliferation requires the localization of PKA to the cilium base. J. Cell Sci. 123, 62-69 (2010).

9. Santos, N. \& Reiter, J. F. A central region of $\mathrm{Gli2}$ regulates its localization to the primary cilium and transcriptional activity. J. Cell Sci. 127, 1500-1510 (2014).

10. Haycraft, C. J. et al. Gli2 and Gli3 Localize to Cilia and Require the Intraflagellar Transport Protein Polaris for Processing and Function. PLOS Genet. 1, e53 (2005).

11. Han, Y. et al. Regulation of Gli ciliary localization and Hedgehog signaling by the PYNLS/karyopherin- $\beta 2$ nuclear import system. PLOS Biol. 15, e2002063 (2017).

12. Lin, Y.-C. et al. Chemically inducible diffusion trap at cilia reveals molecular sieve-like barrier. Nat. Chem. Biol. 9, 437-443 (2013).

13. Nachury, M. V., Seeley, E. S. \& Jin, H. Trafficking to the ciliary membrane: how to get across the periciliary diffusion barrier? Annu. Rev. Cell Dev. Biol. 26, 59-87 (2010).

14. Cassioli, C. \& Baldari, C. T. A Ciliary View of the Immunological Synapse. Cells 8, (2019).

15. Milenkovic, L., Scott, M. P. \& Rohatgi, R. Lateral transport of Smoothened from the plasma membrane to the membrane of the cilium. J. Cell Biol. 187, 365-374 (2009).

16. Monis, W. J., Faundez, V. \& Pazour, G. J. BLOC-1 is required for selective membrane protein trafficking from endosomes to primary cilia. J. Cell Biol. 216, 2131-2150 (2017).

17. Lu, L. \& Madugula, V. Mechanisms of ciliary targeting: entering importins and Rabs. Cell. Mol. Life Sci. CMLS 75, 597-606 (2018).

18. Nassari, S., Del Olmo, T. \& Jean, S. Rabs in Signaling and Embryonic Development. Int. J. Mol. Sci. 21, (2020).

19. Takai, Y., Sasaki, T. \& Matozaki, T. Small GTP-Binding Proteins. Physiol. Rev. 81, 153-208 (2001). 
20. Yoshimura, S.-I., Egerer, J., Fuchs, E., Haas, A. K. \& Barr, F. A. Functional dissection of Rab GTPases involved in primary cilium formation. J. Cell Biol. 178, 363-369 (2007).

21. Kawamura, N. et al. Delivery of endosomes to lysosomes via microautophagy in the visceral endoderm of mouse embryos. Nat. Commun. 3, 1-10 (2012).

22. Singh, S. K., Gui, M., Koh, F., Yip, M. C. \& Brown, A. Structure and activation mechanism of the BBSome membrane protein trafficking complex. eLife $\mathbf{9}$, e53322.

23. Wingfield, J. L., Lechtreck, K.-F. \& Lorentzen, E. Trafficking of ciliary membrane proteins by the intraflagellar transport/BBSome machinery. Essays Biochem. 62, 753-763 (2018).

24. Lobo, G. P. et al. The exocyst is required for photoreceptor ciliogenesis and retinal development. J. Biol. Chem. 292, 14814-14826 (2017).

25. Martin-Urdiroz, M., Deeks, M. J., Horton, C. G., Dawe, H. R. \& Jourdain, I. The Exocyst Complex in Health and Disease. Front. Cell Dev. Biol. 4, 24 (2016).

26. Mei, K. et al. Cryo-EM structure of the exocyst complex. Nat. Struct. Mol. Biol. 25, 139146 (2018).

27. Fogelgren, B. et al. The exocyst protein Sec10 interacts with Polycystin-2 and knockdown causes PKD-phenotypes. PLoS Genet. 7, e1001361 (2011).

28. Zuo, X., Guo, W. \& Lipschutz, J. H. The exocyst protein Sec10 is necessary for primary ciliogenesis and cystogenesis in vitro. Mol. Biol. Cell 20, 2522-2529 (2009).

29. Chen, J. K., Taipale, J., Young, K. E., Maiti, T. \& Beachy, P. A. Small molecule modulation of Smoothened activity. Proc. Natl. Acad. Sci. 99, 14071-14076 (2002).

30. Mellacheruvu, D. et al. The CRAPome: a contaminant repository for affinity purificationmass spectrometry data. Nat. Methods 10, 730-736 (2013).

31. Breslow, D. K. et al. A CRISPR-based screen for Hedgehog signaling provides insights into ciliary function and ciliopathies. Nat. Genet. 50, 460-471 (2018).

32. Han, Y., Shi, Q. \& Jiang, J. Multisite interaction with Sufu regulates Ci/Gli activity through distinct mechanisms in Hh signal transduction. Proc. Natl. Acad. Sci. U. S. A. 112, 6383-6388 (2015).

33. Markiewicz, Ł., Uśpieński, T., Baran, B., Niedziółka, S. M. \& Niewiadomski, P. Xpo7 negatively regulates Hedgehog signaling by exporting Gli2 from the nucleus. Cell. Signal. 80, 109907 (2021).

34. Maurya, A. K. et al. Positive and negative regulation of Gli activity by Kif7 in the zebrafish embryo. PLoS Genet. 9, e1003955 (2013).

35. Ahmed, S. M. et al. Exocyst dynamics during vesicle tethering and fusion. Nat. Commun. 9, 1-17 (2018).

36. Niewiadomski, P. et al. Gli protein activity is controlled by multisite phosphorylation in vertebrate Hedgehog signaling. Cell Rep. 6, 168-181 (2014).

37. Huang, P. et al. Structural Basis of Smoothened Activation in Hedgehog Signaling. Cell 175, 295-297 (2018).

38. Rohatgi, R., Milenkovic, L. \& Scott, M. P. Patched1 regulates hedgehog signaling at the primary cilium. Science $317,372-376$ (2007). 
566

567

568

569

570

571

572

573

574

575

576

577

578

579

580

581

582

583

584

585

586

587

588

589

590

591

592

593

594

595

596

597

598

599

600

601

602

603

604

605

606

39. Luo, G., Zhang, J. \& Guo, W. The role of Sec3p in secretory vesicle targeting and exocyst complex assembly. Mol. Biol. Cell 25, 3813-3822 (2014).

40. Sung, C.-H. \& Leroux, M. R. The roles of evolutionarily conserved functional modules in cilia-related trafficking. Nat. Cell Biol. 15, 1387-1397 (2013).

41. Lee, S.-H. et al. Export of membrane proteins from the Golgi complex to the primary cilium requires the kinesin motor, KIFC1. FASEB J. Off. Publ. Fed. Am. Soc. Exp. Biol. 32, 957-968 (2018).

42. Leroux, M. R. Taking vesicular transport to the cilium. Cell 129, 1041-1043 (2007).

43. Goldenring, J. R. Recycling endosomes. Curr. Opin. Cell Biol. 35, 117-122 (2015).

44. Naslavsky, N. \& Caplan, S. Endocytic membrane trafficking in the control of centrosome function. Curr. Opin. Cell Biol. 65, 150-155 (2020).

45. Macia, E. et al. Dynasore, a cell-permeable inhibitor of dynamin. Dev. Cell 10, 839-850 (2006).

46. Dutta, D., Williamson, C. D., Cole, N. B. \& Donaldson, J. G. Pitstop 2 is a potent inhibitor of clathrin-independent endocytosis. PloS One 7, e45799 (2012).

47. Miller, S. G., Carnell, L. \& Moore, H. H. Post-Golgi membrane traffic: brefeldin A inhibits export from distal Golgi compartments to the cell surface but not recycling. J. Cell Biol. 118, 267-283 (1992).

48. Kim, J., Kato, M. \& Beachy, P. A. Gli2 trafficking links Hedgehog-dependent activation of Smoothened in the primary cilium to transcriptional activation in the nucleus. Proc. Natl. Acad. Sci. 106, 21666-21671 (2009).

49. Stenmark, H. Rab GTPases as coordinators of vesicle traffic. Nat. Rev. Mol. Cell Biol. 10, 513-525 (2009).

50. Lin, F. et al. Kidney-specific inactivation of the KIF3A subunit of kinesin-II inhibits renal ciliogenesis and produces polycystic kidney disease. Proc. Natl. Acad. Sci. U. S. A. 100, 5286-5291 (2003).

51. Markiewicz, Ł., Uśpieński, T., Niedziółka, S. M. \& Niewiadomski, P. Xpo7 negatively regulates Hedgehog signaling by exporting Gli2 from the nucleus. bioRxiv 2020.01.31.928408 (2020) doi:10.1101/2020.01.31.928408.

52. Umberger, P. A. \& Ogden, S. K. SPOP and CUL3 Modulate the Sonic Hedgehog Signal Response Through Controlled Degradation of GLI Family Transcription Factors. Front. Cell Dev. Biol. 9, 710295 (2021).

53. Eggenschwiler, J. T., Bulgakov, O. V., Qin, J., Li, T. \& Anderson, K. V. Mouse Rab23 regulates hedgehog signaling from smoothened to Gli proteins. Dev. Biol. 290, 1-12 (2006).

54. Hor, C., Lo, J., Cham, A., Leong, W. Y. \& Goh, E. Multifaceted functions of Rab23 on primary cilium- and Hedgehog signaling-mediated cerebellar granule cell proliferation. J. Neurosci. Off. J. Soc. Neurosci. JN-RM-3005-20 (2021) doi:10.1523/JNEUROSCI.300520.2021.

55. Wang, Y., Ng, E. L. \& Tang, B. L. Rab23: what exactly does it traffic? Traffic Cph. Den. 7, 746-750 (2006). 
56. Das, A. \& Guo, W. Rabs and the exocyst in ciliogenesis, tubulogenesis and beyond. Trends Cell Biol. 21, 383-386 (2011).

57. Feng, S. et al. A Rab8 guanine nucleotide exchange factor-effector interaction network regulates primary ciliogenesis. J. Biol. Chem. 287, 15602-15609 (2012).

58. Lee, K. H. et al. Identification of a novel Wnt5a-CK1ع-Dvl2-Plk1-mediated primary cilia disassembly pathway. EMBO J. 31, 3104-3117 (2012).

59. Morris, R. L. et al. Redistribution of the kinesin-II subunit KAP from cilia to nuclei during the mitotic and ciliogenic cycles in sea urchin embryos. Dev. Biol. 274, 56-69 (2004).

60. Viau, A. et al. Cilia-localized LKB1 regulates chemokine signaling, macrophage recruitment, and tissue homeostasis in the kidney. EMBO J. 37, (2018).

61. Clement, D. L. et al. PDGFR $\alpha$ signaling in the primary cilium regulates NHE1-dependent fibroblast migration via coordinated differential activity of MEK1/2-ERK1/2-p90RSK and AKT signaling pathways. J. Cell Sci. 126, 953-965 (2013).

62. Peixoto, E. et al. HDAC6-dependent ciliophagy is involved in ciliary loss and cholangiocarcinoma growth in human cells and murine models. Am. J. Physiol. Gastrointest. Liver Physiol. 318, G1022-G1033 (2020).

63. Lai, Y. \& Jiang, Y. Reciprocal Regulation between Primary Cilia and mTORC1. Genes 11, E711 (2020).

64. Emechebe, U. et al. T-box3 is a ciliary protein and regulates stability of the Gli3 transcription factor to control digit number. eLife 5, e07897 (2016).

65. Raman, M. et al. Systematic VCP-UBXD Adaptor Network Proteomics Identifies a Role for UBXN10 in Regulating Ciliogenesis. Nat. Cell Biol. 17, 1356-1369 (2015).

66. Picco, A. et al. The In Vivo Architecture of the Exocyst Provides Structural Basis for Exocytosis. Cell 168, 400-412.e18 (2017).

67. Wood, C. R. \& Rosenbaum, J. L. Proteins of the ciliary axoneme are found on cytoplasmic membrane vesicles during growth of cilia. Curr. Biol. CB 24, 1114-1120 (2014).

68. Nachury, M. V. \& Mick, D. U. Establishing and regulating the composition of cilia for signal transduction. Nat. Rev. Mol. Cell Biol. 20, 389-405 (2019).

69. Pedersen, L. B., Veland, I. R., Schrøder, J. M. \& Christensen, S. T. Assembly of primary cilia. Dev. Dyn. Off. Publ. Am. Assoc. Anat. 237, 1993-2006 (2008).

70. Reed, S. E. et al. A role for Rab14 in the endocytic trafficking of GLUT4 in 3T3-L1 adipocytes. J. Cell Sci. 126, 1931-1941 (2013).

71. Proikas-Cezanne, T., Gaugel, A., Frickey, T. \& Nordheim, A. Rab14 is part of the early endosomal clathrin-coated TGN microdomain. FEBS Lett. 580, 5241-5246 (2006).

72. Junutula, J. R. et al. Rab14 Is Involved in Membrane Trafficking between the Golgi Complex and Endosomes. Mol. Biol. Cell 15, 2218-2229 (2004).

73. Ueno, H., Huang, X., Tanaka, Y. \& Hirokawa, N. KIF16B/Rab14 Molecular Motor Complex Is Critical for Early Embryonic Development by Transporting FGF Receptor. Dev. Cell 20, 60-71 (2011). 
74. Lu, R. \& Wilson, J. M. Rab14 specifies the apical membrane through Arf6-mediated regulation of lipid domains and Cdc42. Sci. Rep. 6, (2016).

75. Dejgaard, S. Y. et al. Rab18 and Rab43 have key roles in ER-Golgi trafficking. J. Cell Sci. 121, 2768-2781 (2008).

76. Martin, S. \& Parton, R. G. Characterization of Rab18, a lipid droplet-associated small GTPase. Methods Enzymol. 438, 109-129 (2008).

77. Li, C. et al. COPI-TRAPPII activates Rab18 and regulates its lipid droplet association. EMBO J. 36, 441-457 (2017).

78. Loukil, A., Barrington, C. \& Goetz, S. C. A complex of distal appendage-associated kinases linked to human disease regulates ciliary trafficking and stability. Proc. Natl. Acad. Sci. 118, (2021).

79. Leaf, A. \& Von Zastrow, M. Dopamine receptors reveal an essential role of IFT-B, KIF17, and Rab23 in delivering specific receptors to primary cilia. elife 4, e06996 (2015).

80. Boehlke, C. et al. Differential role of Rab proteins in ciliary trafficking: Rab23 regulates smoothened levels. J. Cell Sci. 123, 1460-1467 (2010).

81. Lim, Y. S. \& Tang, B. L. A role for Rab23 in the trafficking of Kif17 to the primary cilium. J. Cell Sci. 128, 2996-3008 (2015).

82. Evans, T. M., Ferguson, C., Wainwright, B. J., Parton, R. G. \& Wicking, C. Rab23, a negative regulator of hedgehog signaling, localizes to the plasma membrane and the endocytic pathway. Traffic Cph. Den. 4, 869-884 (2003).

83. Follit, J. A. et al. Arf4 is required for Mammalian development but dispensable for ciliary assembly. PLoS Genet. 10, e1004170 (2014).

84. Gillingham, A. K. \& Munro, S. The small G proteins of the Arf family and their regulators. Annu. Rev. Cell Dev. Biol. 23, 579-611 (2007).

85. Deretic, D., Lorentzen, E. \& Fresquez, T. The ins and outs of the Arf4-based ciliary membrane-targeting complex. Small GTPases 1-12 (2019) doi:10.1080/21541248.2019.1616355.

86. Oguchi, M. E., Okuyama, K., Homma, Y. \& Fukuda, M. A comprehensive analysis of Rab GTPases reveals a role for Rab34 in serum starvation-induced primary ciliogenesis. J. Biol. Chem. 295, 12674-12685 (2020).

87. Westlake, C. J. et al. Primary cilia membrane assembly is initiated by Rab11 and transport protein particle II (TRAPPII) complex-dependent trafficking of Rabin8 to the centrosome. Proc. Natl. Acad. Sci. 108, 2759-2764 (2011).

88. Grozdanov, P. N. \& MacDonald, C. C. Generation of plasmid vectors expressing FLAGtagged proteins under the regulation of human elongation factor-1 $\alpha$ promoter using Gibson assembly. J. Vis. Exp. JoVE (2015) doi:10.3791/52235.

89. Martin, T. D. et al. Ral and Rheb GTPase activating proteins integrate mTOR and GTPase signaling in aging, autophagy, and tumor cell invasion. Mol. Cell 53, 209-220 (2014).

90. Bindels, D. S. et al. mScarlet: a bright monomeric red fluorescent protein for cellular imaging. Nat. Methods 14, 53-56 (2017). 
91. Moffat, J. et al. A lentiviral RNAi library for human and mouse genes applied to an arrayed viral high-content screen. Cell 124, 1283-1298 (2006).

92. Bryant, D. M. et al. A molecular network for de novo generation of the apical surface and lumen. Nat. Cell Biol. 12, 1035-1045 (2010).

93. Sanjana, N. E., Shalem, O. \& Zhang, F. Improved vectors and genome-wide libraries for CRISPR screening. Nat. Methods 11, 783-784 (2014).

94. Fellmann, C. et al. An optimized microRNA backbone for effective single-copy RNAi. Cell Rep. 5, 1704-1713 (2013).

95. Dull, T. et al. A third-generation lentivirus vector with a conditional packaging system. J. Virol. 72, 8463-8471 (1998).

96. Huang, B. et al. The Anaplasma phagocytophilum-occupied vacuole selectively recruits Rab-GTPases that are predominantly associated with recycling endosomes. Cell. Microbiol. 12, 1292-1307 (2010).

97. Chun, J., Shapovalova, Z., Dejgaard, S. Y., Presley, J. F. \& Melançon, P. Characterization of class I and II ADP-ribosylation factors (Arfs) in live cells: GDP-bound class II Arfs associate with the ER-Golgi intermediate compartment independently of GBF1. Mol. Biol. Cell 19, 3488-3500 (2008).

98. Nakai, W. et al. ARF1 and ARF4 regulate recycling endosomal morphology and retrograde transport from endosomes to the Golgi apparatus. Mol. Biol. Cell 24, 25702581 (2013).

99. Choudhury, A. et al. Rab proteins mediate Golgi transport of caveola-internalized glycosphingolipids and correct lipid trafficking in Niemann-Pick C cells. J. Clin. Invest. 109, 1541-1550 (2002).

100. Nachury, M. V. et al. A core complex of BBS proteins cooperates with the GTPase Rab8 to promote ciliary membrane biogenesis. Cell 129, 1201-1213 (2007).

101. Humke, E. W., Dorn, K. V., Milenkovic, L., Scott, M. P. \& Rohatgi, R. The output of Hedgehog signaling is controlled by the dynamic association between Suppressor of Fused and the Gli proteins. Genes Dev. 24, 670-682 (2010).

102. Mellacheruvu, D. et al. The CRAPome: a contaminant repository for affinity purificationmass spectrometry data. Nat. Methods 10, 730-736 (2013).

103. $\mathrm{Mi}, \mathrm{H}$. et al. PANTHER version 16 : a revised family classification, tree-based classification tool, enhancer regions and extensive API. Nucleic Acids Res. 49, D394D403 (2021).

104. Doench, J. G. et al. Optimized sgRNA design to maximize activity and minimize offtarget effects of CRISPR-Cas9. Nat. Biotechnol. 34, 184-191 (2016).

105. Söderberg, O. et al. Direct observation of individual endogenous protein complexes in situ by proximity ligation. Nat. Methods 3, 995-1000 (2006).

106. Szklarczyk, D. et al. STRING v11: protein-protein association networks with increased coverage, supporting functional discovery in genome-wide experimental datasets. Nucleic Acids Res. 47, D607-D613 (2019). 
bioRxiv preprint doi: https://doi.org/10.1101/2021.09.27.461918; this version posted September 28, 2021. The copyright holder for this preprint (which was not certified by peer review) is the author/funder, who has granted bioRxiv a license to display the preprint in perpetuity. It is made available under aCC-BY 4.0 International license.

727 107. Goodsell, D. S., Autin, L. \& Olson, A. J. Illustrate: Software for Biomolecular Illustration.

728 Structure 27, 1716-1720.e1 (2019).

729

730 
bioRxiv preprint doi: https://doi.org/10.1101/2021.09.27.461918; this version posted September 28, 2021. The copyright holder for this

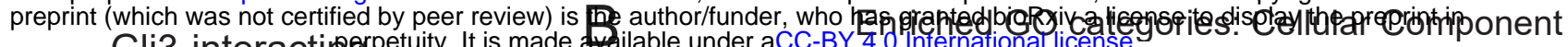

Gli3-interacti甲egrpetuity. It is made ablable under aCC-BY 4.0 International license. proteins

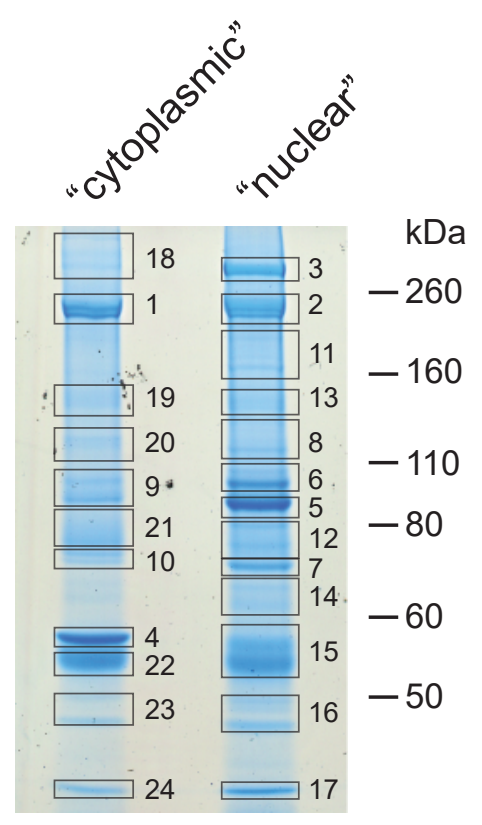

C
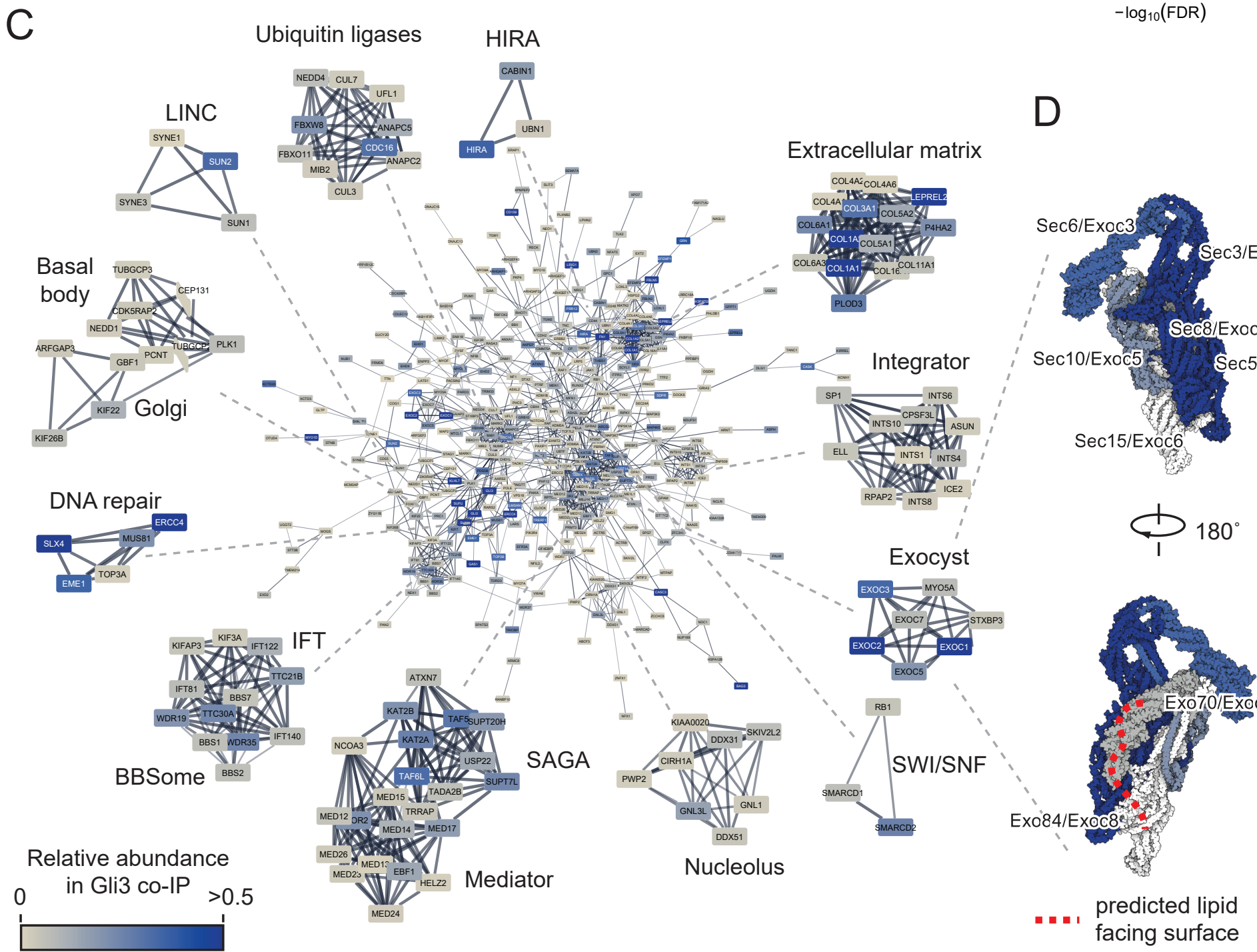

Ubiquitin ligases

HIRA
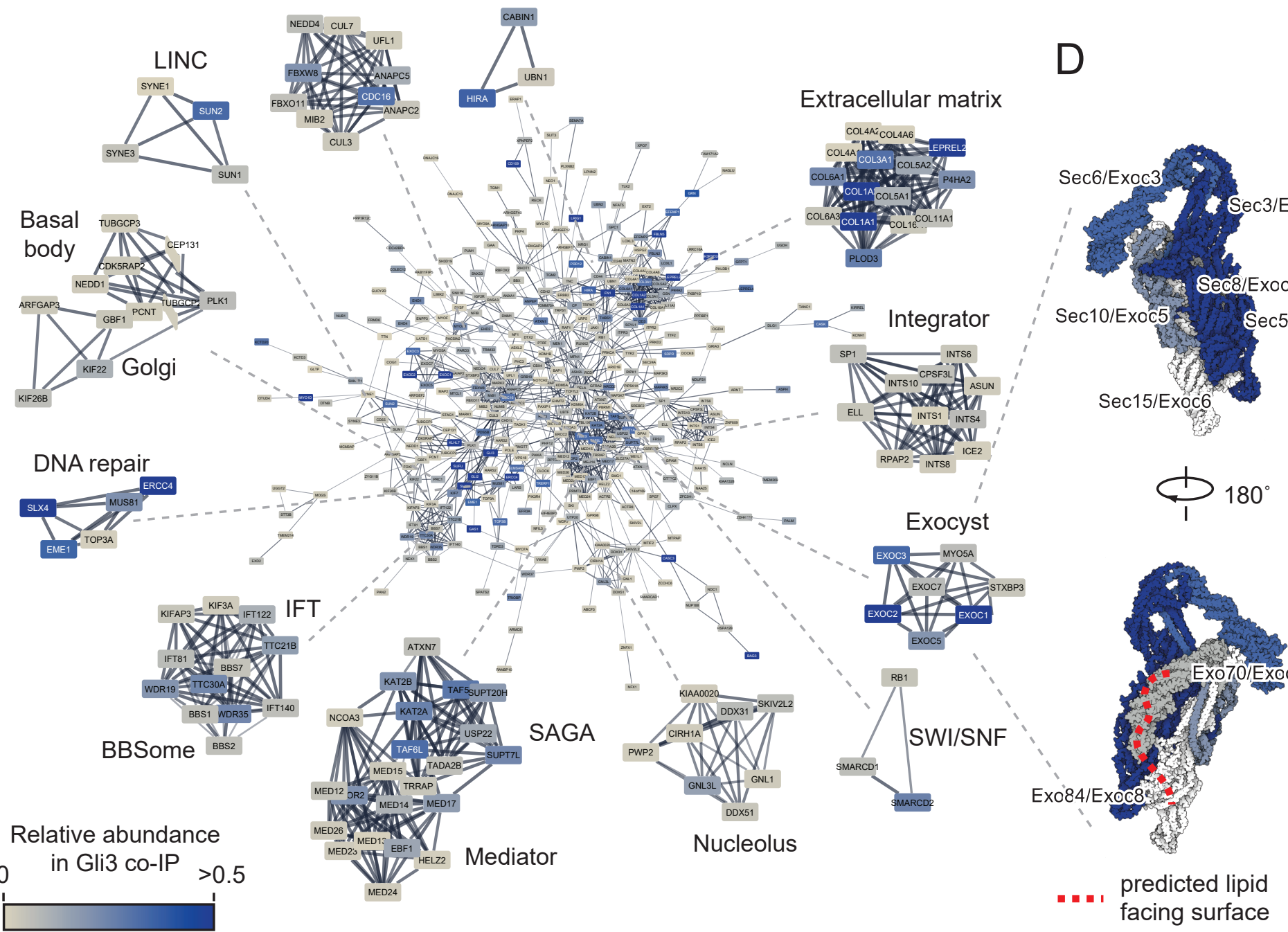

collagen trimer (GO:0005581)

mediator complex (GO:0016592)

core mediator complex (GO:0070847)

SAGA-type complex (GO:0070461)

exocyst (GO:0000145)

SWI/SNF superfamily-type complex (GO:0070603)

supramolecular polymer (GO:0099081)

ciliary basal body (GO:0036064)

BBSome (GO:0034464)

integrator complex (GO:0032039)

gamma-tubulin complex (GO:0000930)

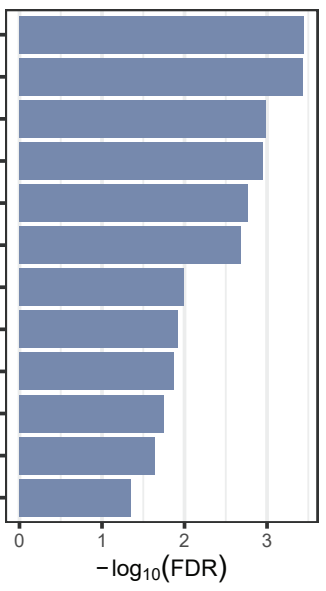

Enriched GO categories: Biological Process

extracellular matrix organization (GO:0030198) intraciliary retrograde transport (GO:0035721)

chromatin remodeling (GO:0006338) peptidyl-amino acid modification (GO:0018193) microtubule bundle formation (GO:0001578) histone modification (GO:0016570) membrane fusion (GO:0061025)

$G$ protein-coupled receptor signaling pathway (GO:0007186)
gene expression (GO:0010467)

G protein-coupled receptor signaling pathway (GO:0007186)
gene expression (GO:0010467) cellular macromolecule biosynthetic process (GO:0034645) resolution of meiotic recombination intermediates (GO:0000712)
mitotic nuclear division (GO:0140014) vesicle targeting, to, from or within Golgi (GO:0048199) RNA biosynthetic process (GO:0032774) protein localization to cilium (GO:0061512) DNA repair (GO:0006281) protein acylation (GO:0043543)

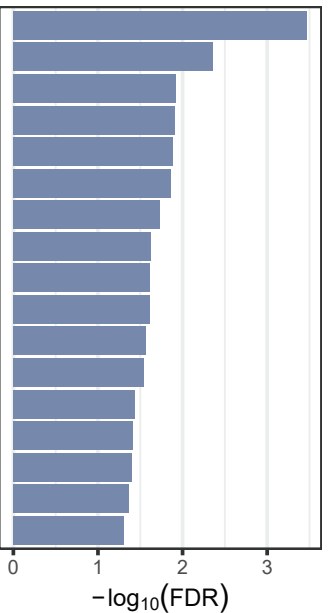


Fig. 1 Gli3 interactome is enriched for proteins involved in ciliary transport of vesicles

(A) NIH/3T3 Flp-In cells were treated with 100nM SAG for 4h, roughly fractionated into a "cytoplasmic" and "nuclear" fraction, and each fraction was pulled down using magnetic beads coated with the anti-Gli3 antibody. Proteins were resolved on SDS-PAGE, the gel was stained with coomassie brilliant blue and prominent bands were excised for mass spectrometry-based protein ID. Shown is the image of the coomassie-stained gel with each of the excised bands indicated and numbered. Gli3 is enriched in bands 1 ("cytoplasmic") and 2 ("nuclear"). (B) MS-identified proteins from all bands were pooled and common MS-AP contaminants (>10\% FDR from the CRAPome database 30) were removed. PANTHER 103 was used to find overrepresented Gene Ontology (GO) terms in the "PANTHER GO - Slim Biological Process" and "PANTHER GO - Slim Cellular Component" categories. Top-level enriched GO terms are shown with their corresponding -log10(FDR) values. (C) High confidence Gli3 interactors identified by MS were connected into a network using the STRING 106 plugin in Cytoscape. Shown is the main protein network with the node color representing the approximate relative abundance of the protein in the Gli3 interactome and the edge thickness corresponding to the confidence of connection between proteins in the STRING database. Also shown are highly interconnected sub-networks identified using MCODE clustering, which typically corresponds to protein complexes or multiprotein functional units. (D) The exocyst complex structure (PDB ID: 5yfp ref 26) was rendered using Illustrate 107 with each subunit colored according to its abundance in Gli3 IP/MS as in C. Subunits not identified in our experiment are rendered in white. The red dashed line corresponds to the predicted surface of the exocyst complex that comes into contact with the plasma membrane lipids 26. Each subunit is labeled with its alternative gene names. 
bioRxiv preprint doi: https://doi.org/10.1101/2021.09.27.461918; this version posted September 28, 2021. The copyright holder for this

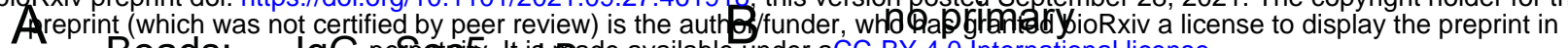

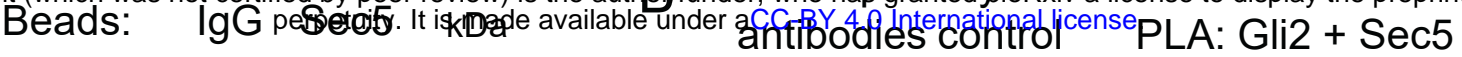
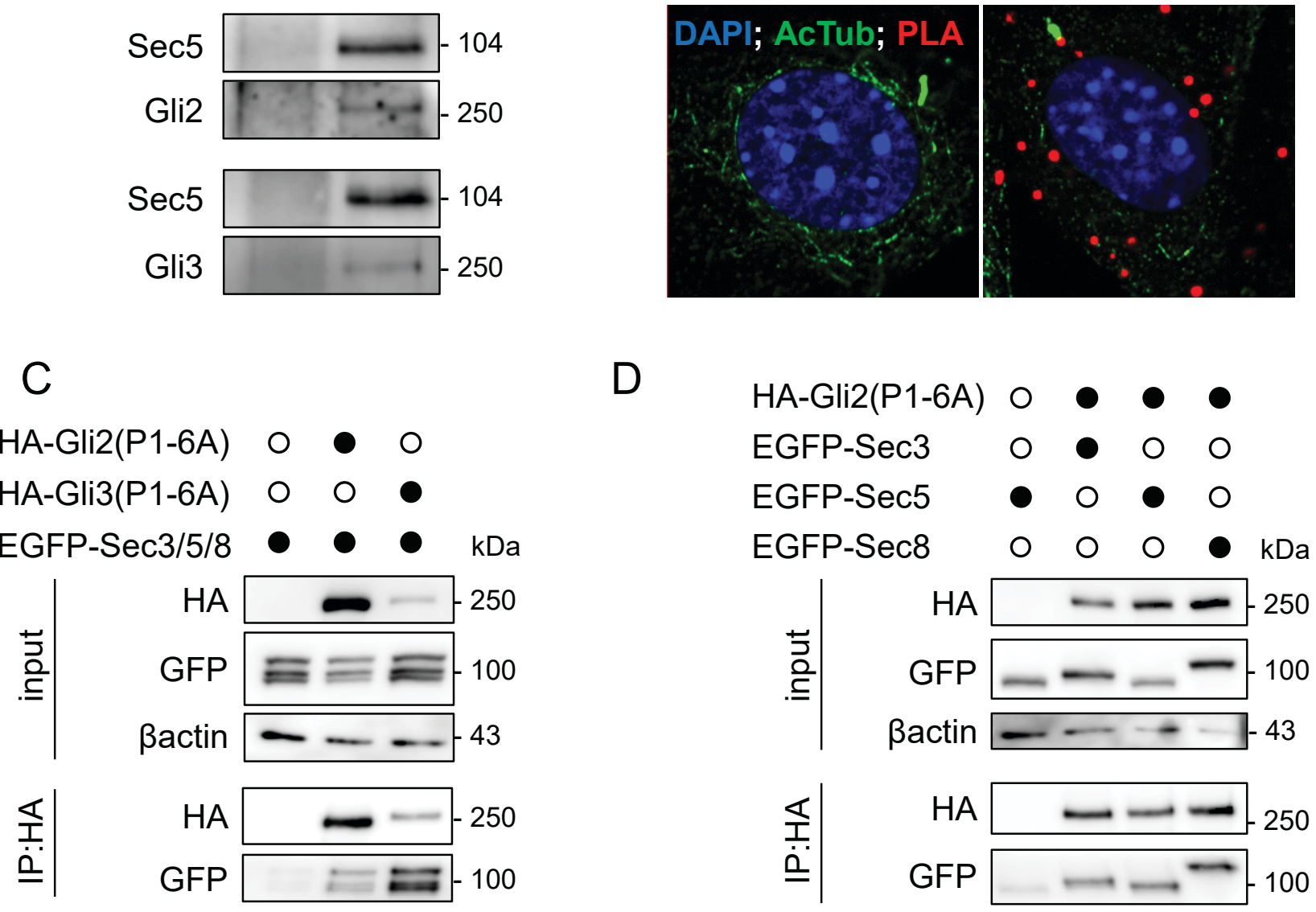

$E$

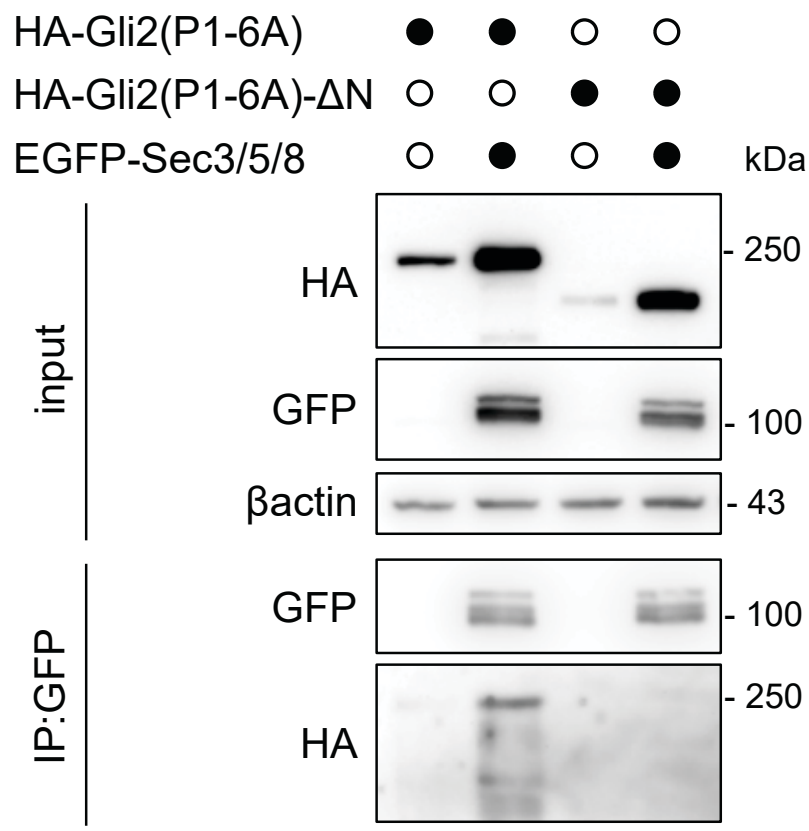

$\mathrm{F}$

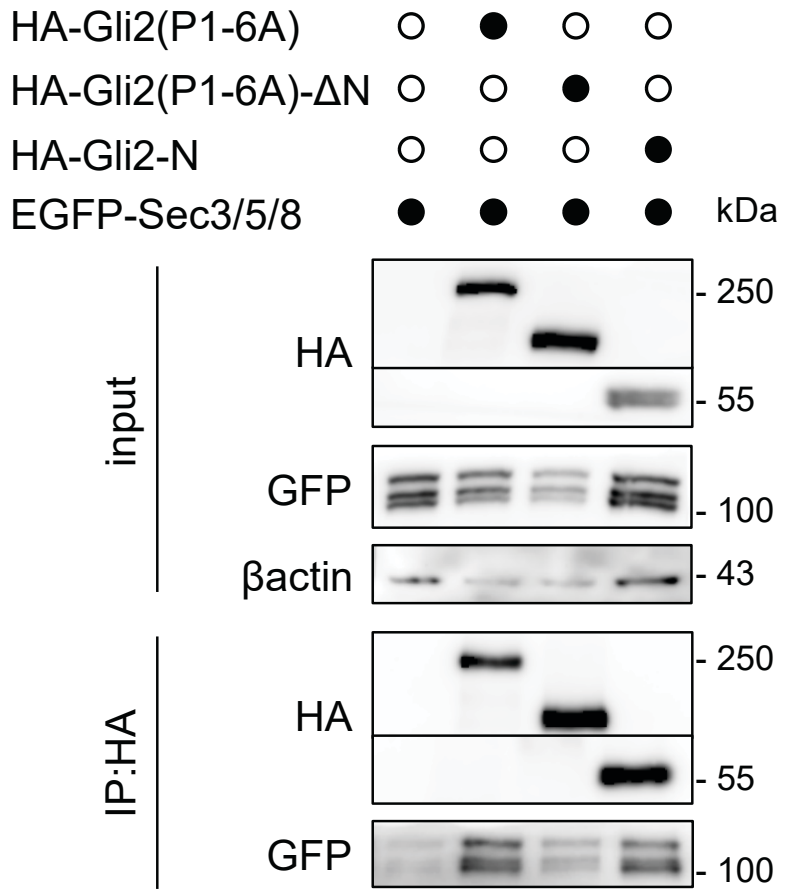

Fig. 2 Exocyst subunits interact with Gli2 and Gli3

(A) Co-immunoprecipitation of endogenous Sec5 with Gli2 and Gli3. Beads were coated with anti-Sec5 antibodies. Rabbit IgG was used as a control. (B) Proximity Ligation Assay with anti-Gli2 and anti-Sec5 antibodies in NIH/3T3 mouse fibroblasts. Sites of interaction are marked in red. Cilia were stained with anti-acetylated tubulin (green), and nuclei with DAPI (blue) (C) Co-immunoprecipitation of overexpressed HA-Gli2(P1-6A) and HA-Gli3(P1-6A) with the exocyst subunits Sec3, Sec5, and Sec8 tagged with EGFP in HEK293T cells using anti-HA beads (D) Co-immunoprecipitation of overexpressed HA-Gli2(P1-6A) with single exocyst subunits Sec3, Sec5 and Sec8 tagged with EGFP in HEK293T cells using anti-HA beads (E) Reciprocal co-immunoprecipitation of overexpressed EGFPtagged Sec3, Sec5 and Sec8 with HA-Gli2(P1-6A) constructs using anti-GFP beads. (F) Co-immunoprecipitation of overexpressed HA-Gli2(P1-6A) truncation constructs with the exocyst subunits Sec3, Sec5, and Sec8 tagged with EGFP in HEK293T cells using anti-HA beads. 
bioRxiv preprint doi: https://doi.org/10.1101/2021.09.27.461918; this version posted September 28, 2021. The copyright holder for this preprint (which was not certified by peer review) is the author/funder, who has granted bioRxiv a license to display the preprint in

A

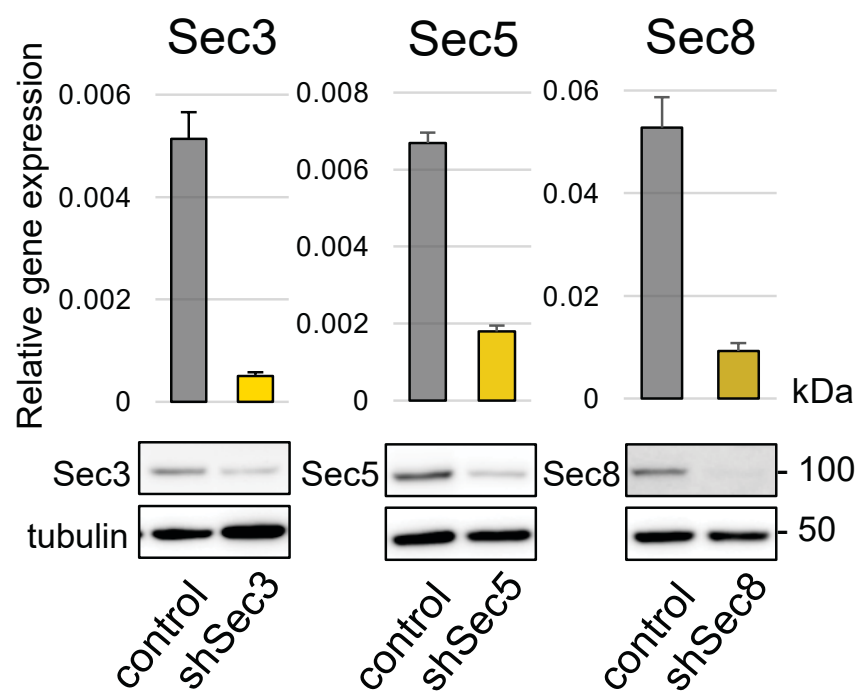
perpetuity. It is made available under aCC-BY 0 International license.

C

$$
\text { Gli2(P1-6A) ciliary localization }
$$

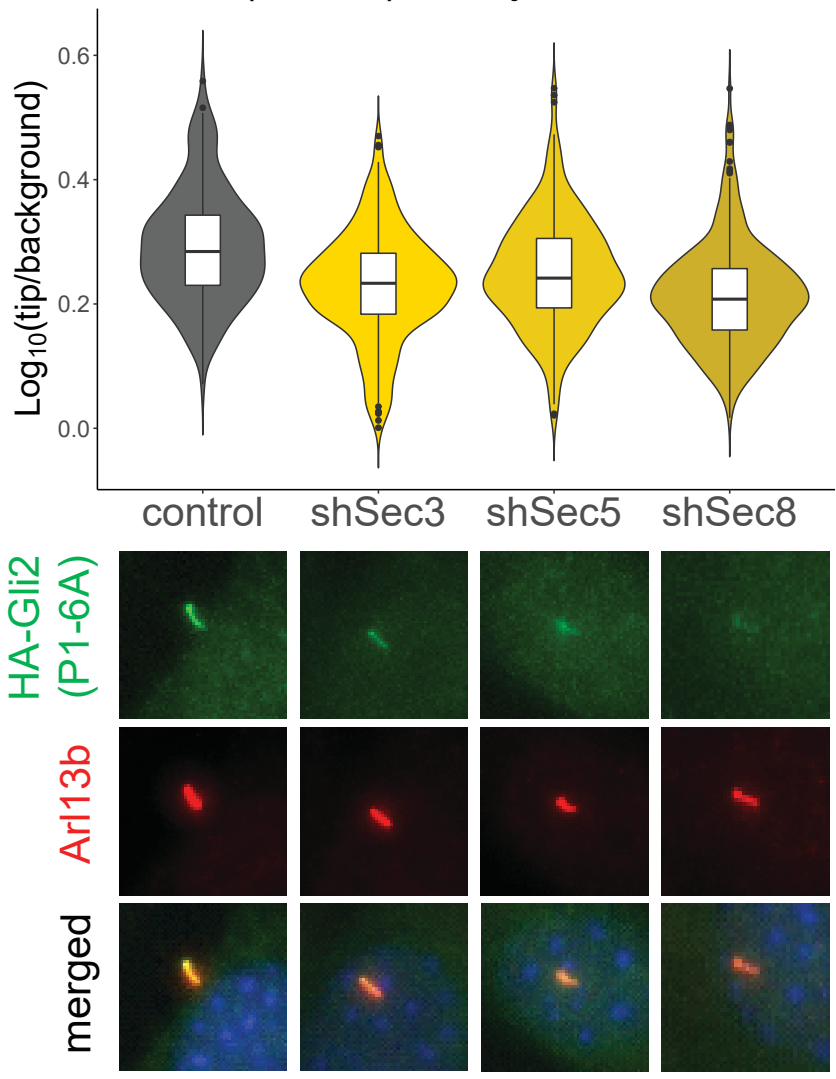

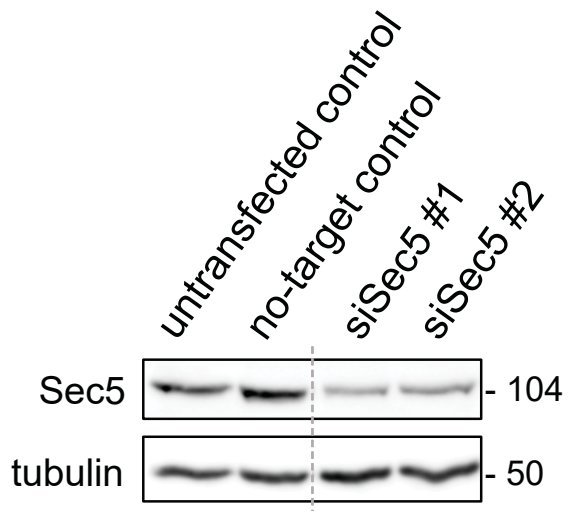

D Gli2(P1-6A) ciliary localization
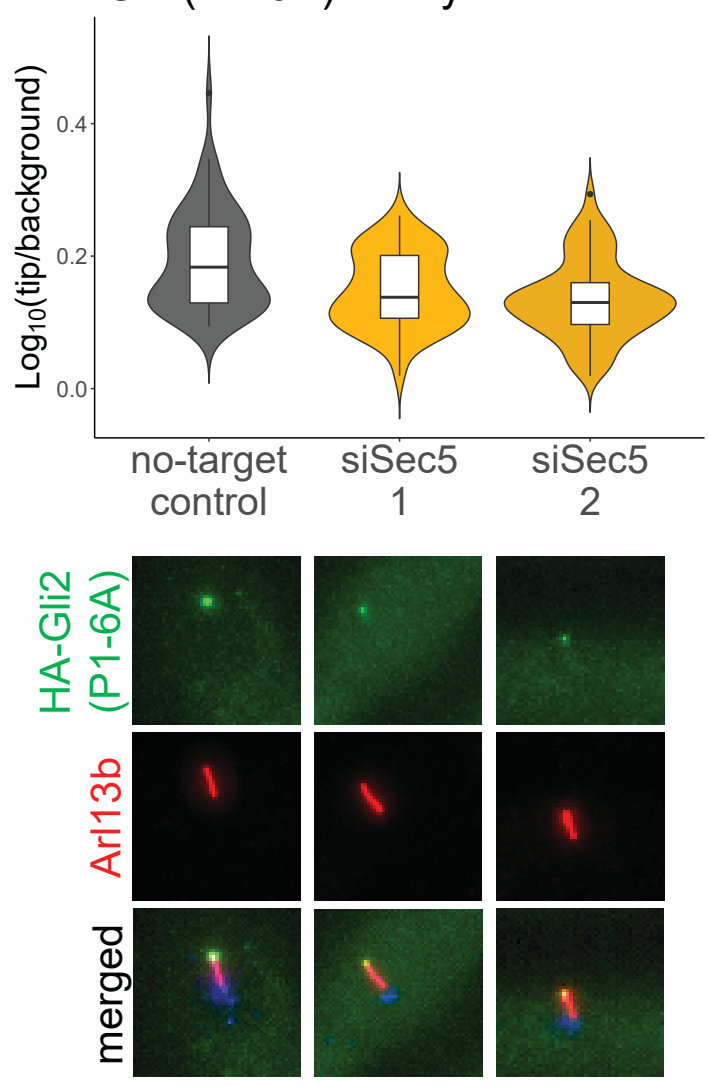

Fig. 3 Knockdown of exocyst subunits decreases Gli2 ciliary localization

(A) mRNA expression levels of the indicated genes in cells stable expressing Gli2(P1-6A) and transduced with shRNA against each of the genes were measured using qRT-PCR. Control cells were transduced with shRNA against luciferase. The protein level of the indicated proteins was detected by western blot. (B) The protein level of Sec5 in cells transfected with siRNA against Sec5 or non-targeting control siRNA. (C) Relative localization at the cilium tip of stably expressed Gli2(P1-6A) in cells with shRNA knockdown of Sec3, Sec5, and Sec8. Results are presented as violin plots of log-transformed ratios of fluorescence intensity of anti-HA staining at cilia tips to the intensity in the surrounding background. Cilia per variant $n>170$. Student's t-test analysis control-shSec3 $p$-value $=$ 5.399e-12; control-shSec5 p-value $=2.206 \mathrm{e}-06$; control-shSec8 p-value < 2.2e-16. Representative images of Gli2(P1-6A) ciliary localization for each condition are presented below. Arl13b was used as a ciliary marker. (D) Relative localization at the cilium tip of Gli2(P1-6A) in cells transfected with indicated siRNAs. Fluorescence intensities were quantified as in Fig. $3 \mathrm{C}$ from $n>60$ cilia per group. Student's t-test for no-target control-siRNA2 $p$-value = 0.0001015 ; for no-target control-siRNA3 p-value $=1.581 \mathrm{e}-06$. Representative images of Gli2(P1-6A) ciliary localization for each condition are presented below. Arl13b was used as a ciliary marker and pericentrin (blue) as a basal body marker. 


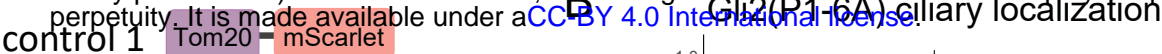

control 2

$\mathrm{mScarlet}=\operatorname{Sec} 5$

mitochondrial trap Tom20-mScarlet - Sec5
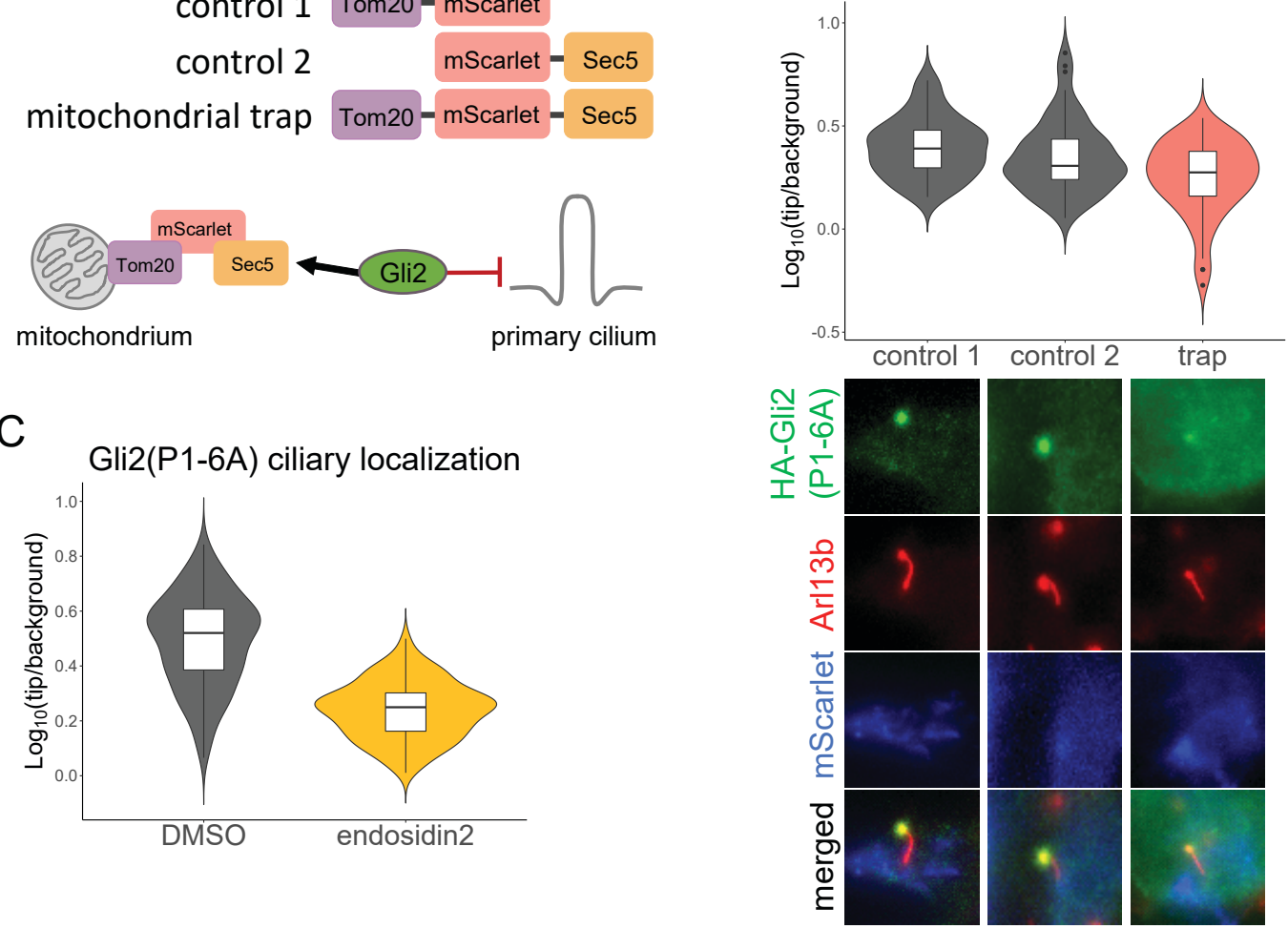

C

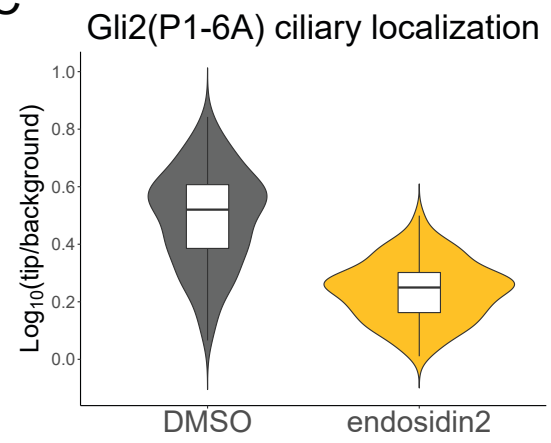

$E$
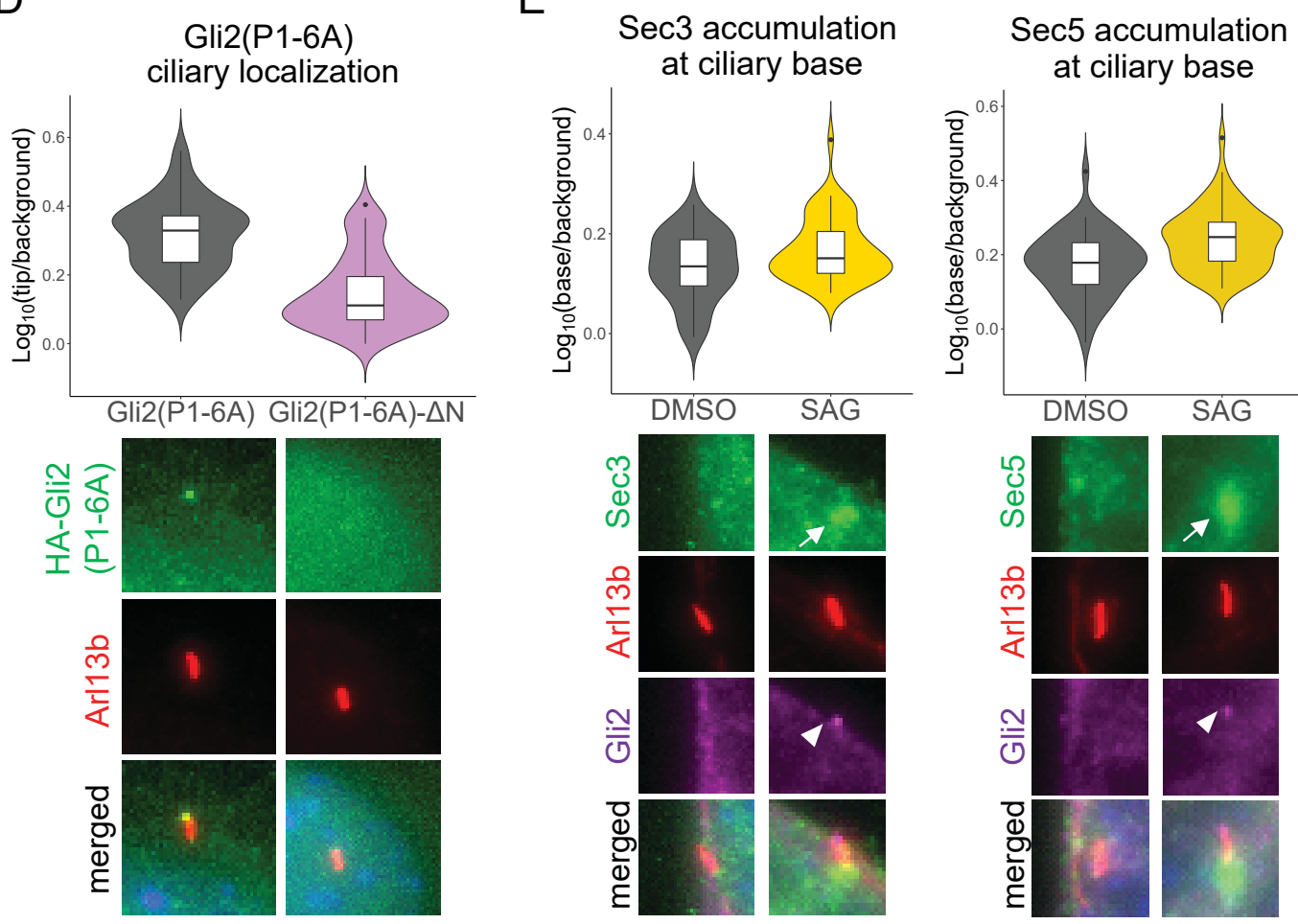

Fig. 4 Impairment of exocyst function reduces Gli2 ciliary localization

(A) Schematic representation of the exocyst mitochondrial trap constructs (top) and assay (bottom) (B) Relative localization at the cilium tip of Gli2(P1-6A) in HEK293T cells co-transfected with the HA-Gli2(P1-6A) and the indicated constructs in $n>40$ cilia. Fluorescence intensities were quantified as in Fig. 3C. Student's test for control 1 vs trap $\mathrm{p}$-value $=4.28 \mathrm{e}-06$; control 2 vs. trap p-value $=0.002$. Representative images of Gli2(P1-6A) ciliary localization are presented below. $(\mathrm{C})$ The exocyst inhibitor endosidin2 blocks the ciliary accumulation of Gli2(P1-6A). Relative localiza-

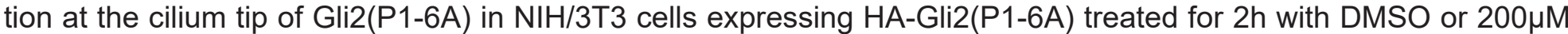
endosidin2 was measured in $n>100$ cilia per group. Fluorescence intensities were quantified as in Fig. $3 \mathrm{C}$. Student's t-test $p$-value $<2.2 e-16$ (D) Gli2(P1-6A)- $\Delta \mathrm{N}$ is largely excluded from the tip of cilia. Relative localization at the cilium tip of Gli2 constructs stably expressed in NIH/3T3 cells. Fluorescence intensities were quantified as in Fig. $3 \mathrm{C}$ in $n>$ 50 cilia per group. Student's t-test p-value $=2.2 \mathrm{e}-14$. Representative images are presented below. Arl13b was used as a ciliary marker. (E) Effect of Smoothened agonist (SAG) treatment (24h; 200nM) on the accumulation of Sec3 and Sec5 at the ciliary base in NIH/3T3 cells. Cells were stained with anti-Sec3 or anti-Sec5 and the ciliary marker acetylated $\alpha$-tubulin (AcTub). Relative localization at the cilium base was measured in $n>40$ cells per group as in Fig. 3C. Student's t-test Sec3: control vs SAG p-value $=0.005$; Sec5: control vs SAG p-value $=4.6 e-05$. Representative images for each condition are presented below. Arl13b was used as a ciliary marker. White arrows show Sec3/5 accumulation and white arrowheads show Gli2 ciliary accumulation. 
bioRxiv preprint doi: https://doi.org/10.1101/2021.09.27.461918; this version posted September 28, 2021. The copyright holder for this preprint (which was not certified by peer review) is the author/funder, who has granted bioRxiv a license to display the preprint in perpetuity. It is made available under aCC-BY 4.0 International license.

A

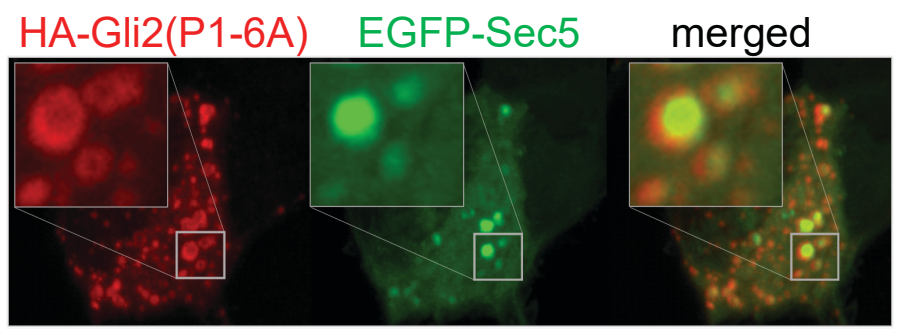

C

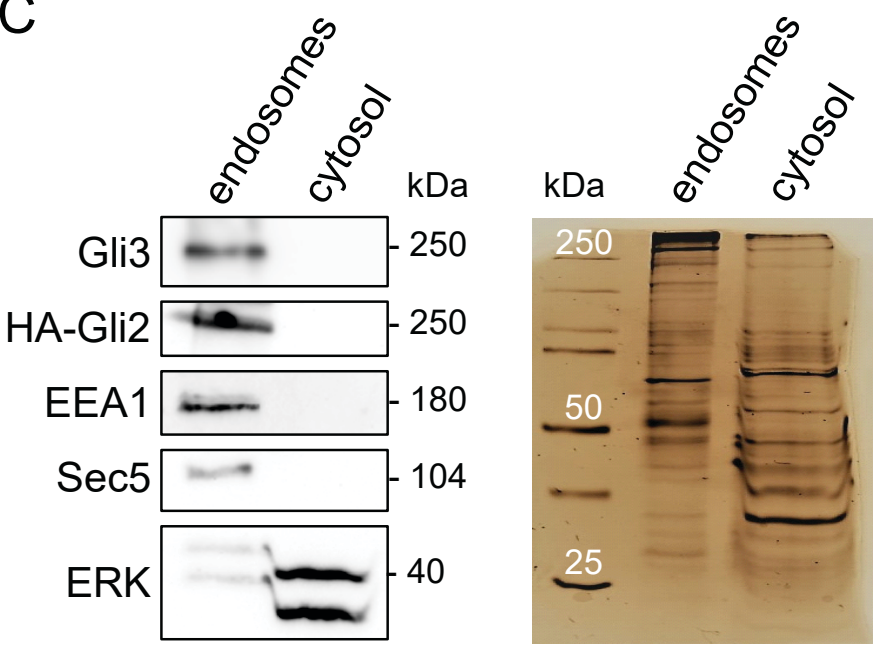

E

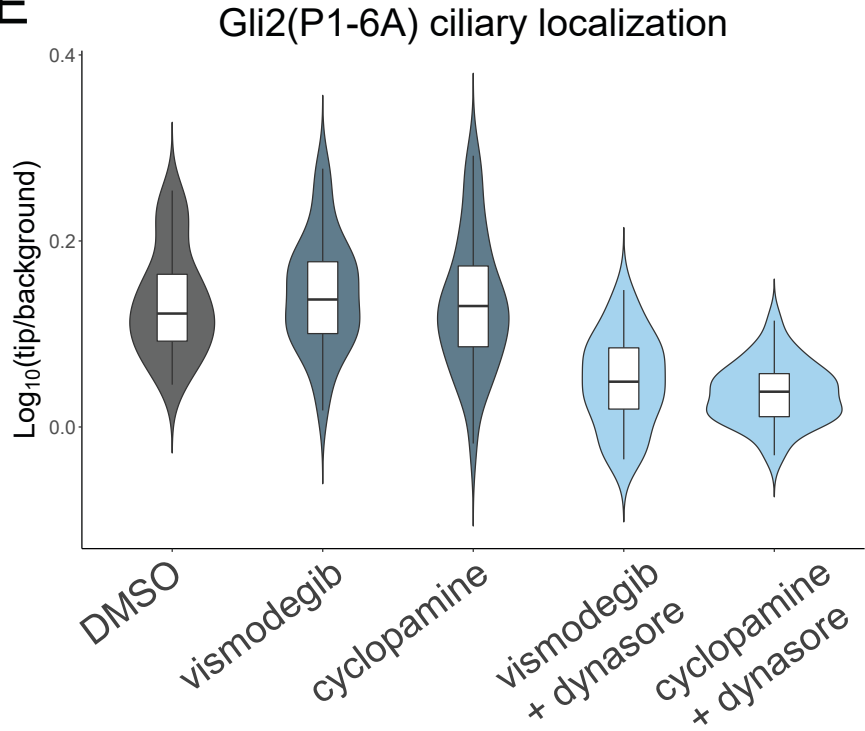

$\mathrm{H}$

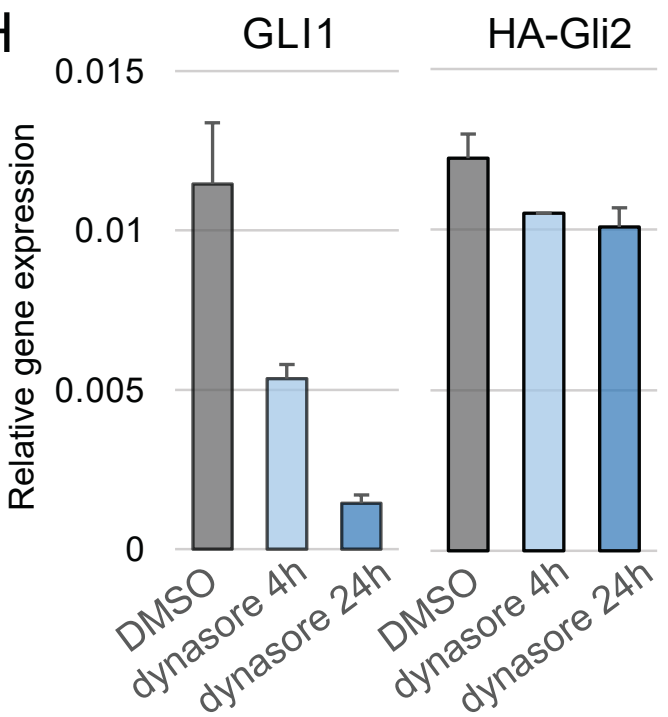

B
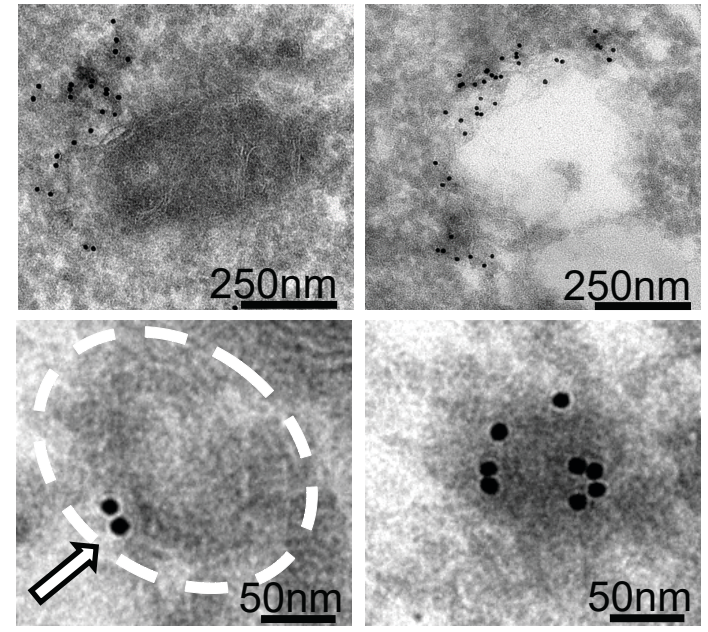

D
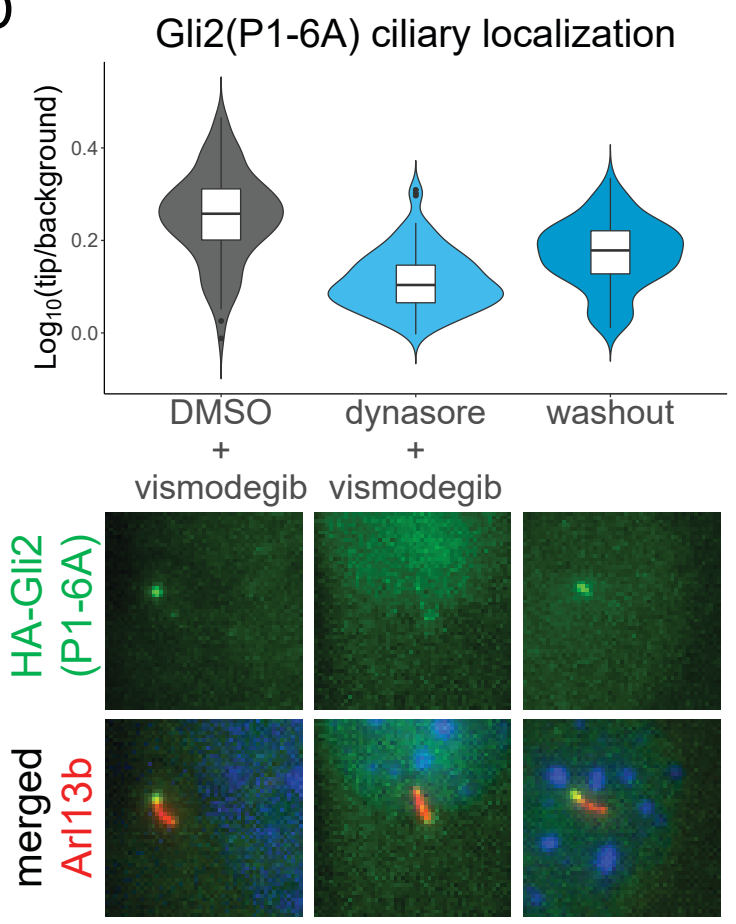

F Gli2(P1-6A) ciliary localization

G Gli2(P1-6A) ciliary localization
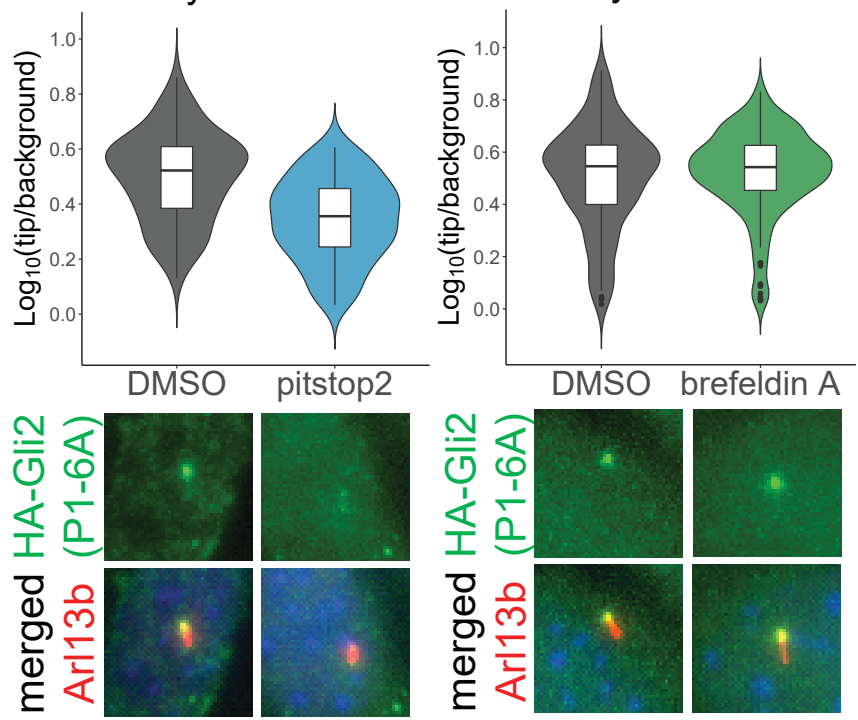
bioRxiv preprint doi: https://doi.org/10.1101/2021.09.27.461918; this version posted September 28, 2021. The copyright holder for this preprint (which was not certified by peer review) is the author/funder, who has granted bioRxiv a license to display the preprint in perpetuity. It is made available under aCC-BY 4.0 International license.

Fig. 5 Gli2 associates with intracellular vesicles

(A) Airyscan fluorescence imaging of HEK293T cells co-transfected with HA-Gli2(P1-6A) and EGFP-Sec5 stained with anti-HA. Insets show high magnification of the Sec5- and HA-Gli2(P1-6A)-positive vesicle-like structures with Gli2 at the periphery (B) Electron microscopy images of HEK293T cells transfected with EGFP-Gli2(P1-6A) and labeled with immunogold-conjugated anti-GFP. EGFP-positive signal accumulates around vesicle-like structures (C) Cells stably expressing HA-Gli2(P1-6A) were fractionated using the endosome isolation kit and the fractions were resolved using SDS-PAGE. Immunoblot shows HA-Gli2(P16A), Gli3, and Sec5 in the endosomal fraction. EEA1 was used as a marker of the endosomes, and ERK was used as a cytosolic fraction marker. The same protein samples were resolved by SDS-PAGE and the gel was silver-stained, showing similar total protein abundance in both fractions. (D) Dynasore impairs Gli2(P1-6A) ciliary localization. Cells were treated with vismodegib in the presence or absence of dynasore for $2 \mathrm{~h}$ hours and then the drugs were washed out for the indicated times. Relative localization of Gli2(P1$6 \mathrm{~A}$ ) at the cilium tip was measured as in Fig. $3 \mathrm{C}$ for $\mathrm{n}>80$ cilia per group. Student's t-test DMSO+vismodegib vs dynasore+vismodegib p-value < 2.2e-16; dynasore+vismodegib vs washout $1 \mathrm{~h}$ $\mathrm{p}$-value $=4.25 \mathrm{e}-05$; dynasore+vismodegib vs washout $4 \mathrm{~h} \mathrm{p}$-value $=6.412 \mathrm{e}-10$. Representative images of Gli2(P1-6A) ciliary localization for each condition are presented below. Arl13b was used as a ciliary marker. (E) Effect of dynasore treatment on Gli2(P1-6A) ciliary accumulation. NIH/3T3 cells with stable expression of HA-Gli2(P1-6A) were treated with dynasore $(4 \mathrm{~h} ; 40 \mu \mathrm{M})$ in the presence of Smo inhibitors vismodegib $(4 \mathrm{~h} ; 3 \mu \mathrm{M})$ and cyclopamine $(4 \mathrm{~h} ; 10 \mu \mathrm{M})$. The Smo inhibitors were used to ensure that the effect of dynasore was not due to its influence on Smo or Ptch trafficking. The Smo inhibitors did not influence Gli2(P1-6A) ciliary accumulation, as expected, and did not prevent dynasore from inhibiting Gli2(P1-6A) localization at the cilium tip. Relative localization of Gli2(P1-6A) at the cilium tip was measured as in Fig. $3 C$ for $n>30$ cilia per group. Student's t-test DMSO vs vismodegib $p$-value $=0.5533$; DMSO vs vismodegib+dynasore $p$-value $=9.047 \mathrm{e}-08 ;$ DMSO vs cyclopamine $p$-value $=0.8634 ;$ DMSO vs cyclopamine+dynasore $p$-value $=1.708 \mathrm{e}-10$. $(\mathrm{F})$ Effect of pitstop2 treatment on Gli2(P1-6A) ciliary accumulation. Pitstop2 $(30 \mu \mathrm{M})$ was used for $10 \mathrm{~min}$ and then wash out to avoid its toxicity effect on cell viability. Effect of treatment was observed 30min after washout. Relative localization of Gli2(P1-6A) at the cilium tip was measured as in Fig. $3 \mathrm{C}$ for $n>80$ cilia per group. Student's t-test DMSO vs pitstop2 $30 \mathrm{~min}$ washout $p$-value $=2.486 \mathrm{e}-10$. Representative images of Gli2(P1-6A) ciliary localization for each condition are presented below. Arl13b was used as a ciliary marker. $(G)$ Effect of brefeldin A treatment on Gli2(P1-6A) ciliary accumulation. Cells were treated with DMSO or brefeldin A $(5 \mu \mathrm{g} / \mathrm{ml})$ for $2 \mathrm{~h}$. Relative localization of Gli2(P1-6A) at the cilium tip was measured as in Fig. 3C for $n>140$ cilia per group. Student's t-test DMSO vs brefeldin A p-value $=0.4565$. Representative images of Gli2(P1-6A) ciliary localization for each condition are presented below. Arl13b was used as a ciliary marker. $(\mathrm{H})$ The relative mRNA expression level of Gli1 (Hh pathway activity marker), and HA-Gli2(P1-6A) after $4 \mathrm{~h}$ and $24 \mathrm{~h}$ of dynasore treatment. 


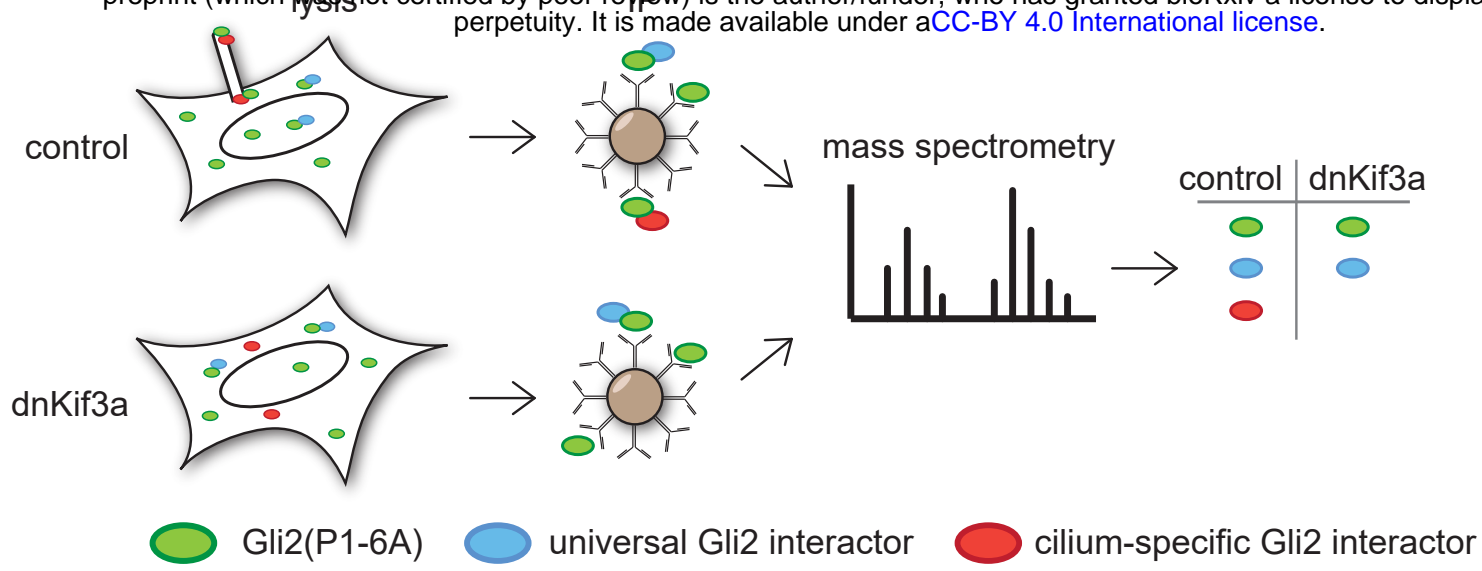

B Gli2 P1-6A-interacting proteins

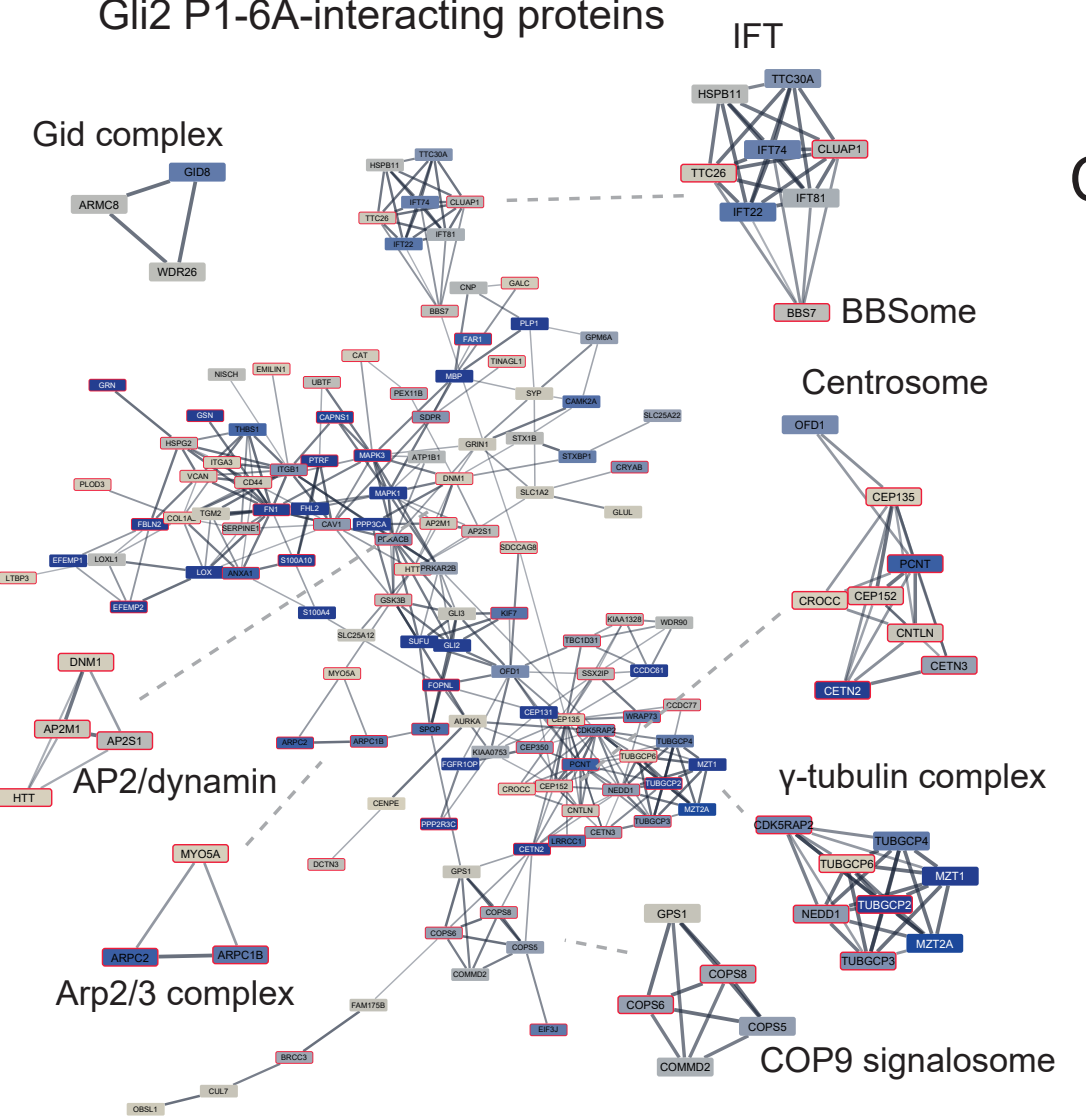

C

Relative abundance in

Enriched in co-IP 0 Gli2 P1-6A co-IP >0.8 from ciliated cells

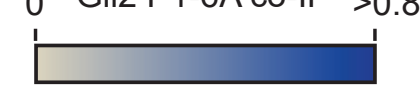

Total Relative co-IP

Protein enrichment in enrichment in FDR Gli2 co-IP ciliated cells

\begin{tabular}{c|c|c|c}
\hline Arf3 & 2.00 & $40 \%$ & $36 \%$ \\
Arf4 & 0.95 & $74 \%$ & $28 \%$ \\
Rab11b & 0.21 & $100 \%$ & $17 \%$ \\
Rab14 & 0.33 & $100 \%$ & $21 \%$ \\
Rab18 & 0.35 & $75 \%$ & $12 \%$ \\
Rab3d & 0.25 & $50 \%$ & $18 \%$ \\
Rab5c & 0.26 & $100 \%$ & $21 \%$
\end{tabular}

Fig. 6 Interaction network of Gli2(P1-6A) in ciliated and non-ciliated cells

(A) Schematic representation of the experiment. NIH/3T3 Flp-In cells stably expressing HA-Gli2(P1-6A) and either vector control or Hdominant-negative Kif3a (dnKif3a) were lysed in gentle lysis buffer and the lysates were immunoprecipitated using magnetic beads coated with anti-HA antibodies. Eluted proteins were submitted for mass spectrometric analysis. Common MS-AP contaminants ( $>10 \%$ FDR from the CRAPome database 30 ) were removed from each dataset (control - ciliated, dnKif3a - non-ciliated) (B) High confidence HA-Gli2(P1-6A) interactors identified by MS were connected into a network using the STRING 106 plugin in Cytoscape. Proteins identified in Gli2(P1-6A) from ciliated and non-ciliated cells were pooled. Shown is the main protein network with the node color representing the approximate relative abundance of the protein in the Gli3 interactome and the edge thickness corresponding to the confidence of connection between proteins in the STRING database. Also shown are highly interconnected subnetworks identified using MCODE clustering, which typically corresponds to protein complexes or multiprotein functional units. Proteins that were identified predominantly in the ciliated cells are marked with red borders (C) Small GTPases identified in Gli2(P1-6A) co-IP/MS experiments are shown, with their relative enrichment scores, relative enrichment in ciliated vs non-ciliated cell co-IP samples, and FDR scores based on the CRAPome database. 
bioRxiv preprint doi: https://doi.org/10.1101/2021.09.27.461918; this version posted September 28, 2021. The copyright holder for this preprint (which was not certified by peer review) is the author/funder, who has granted bioRxiv a license to display the preprint in

A

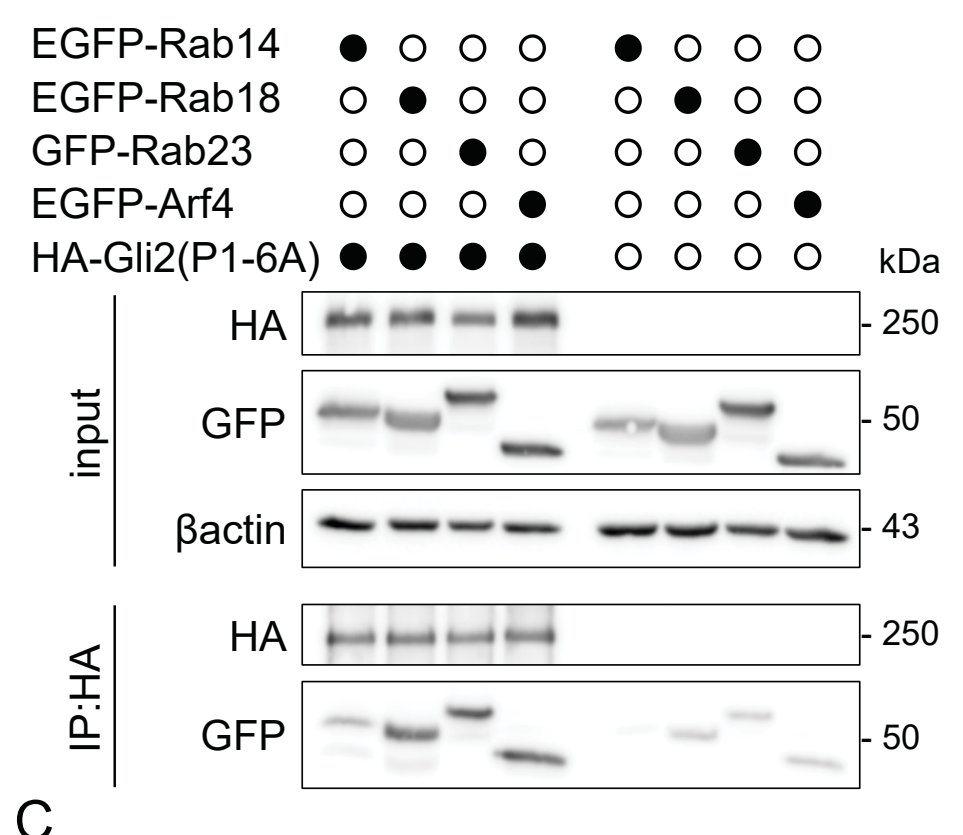

Gli2(P1-6A) ciliary localization

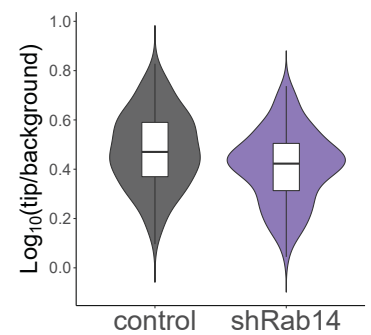

E
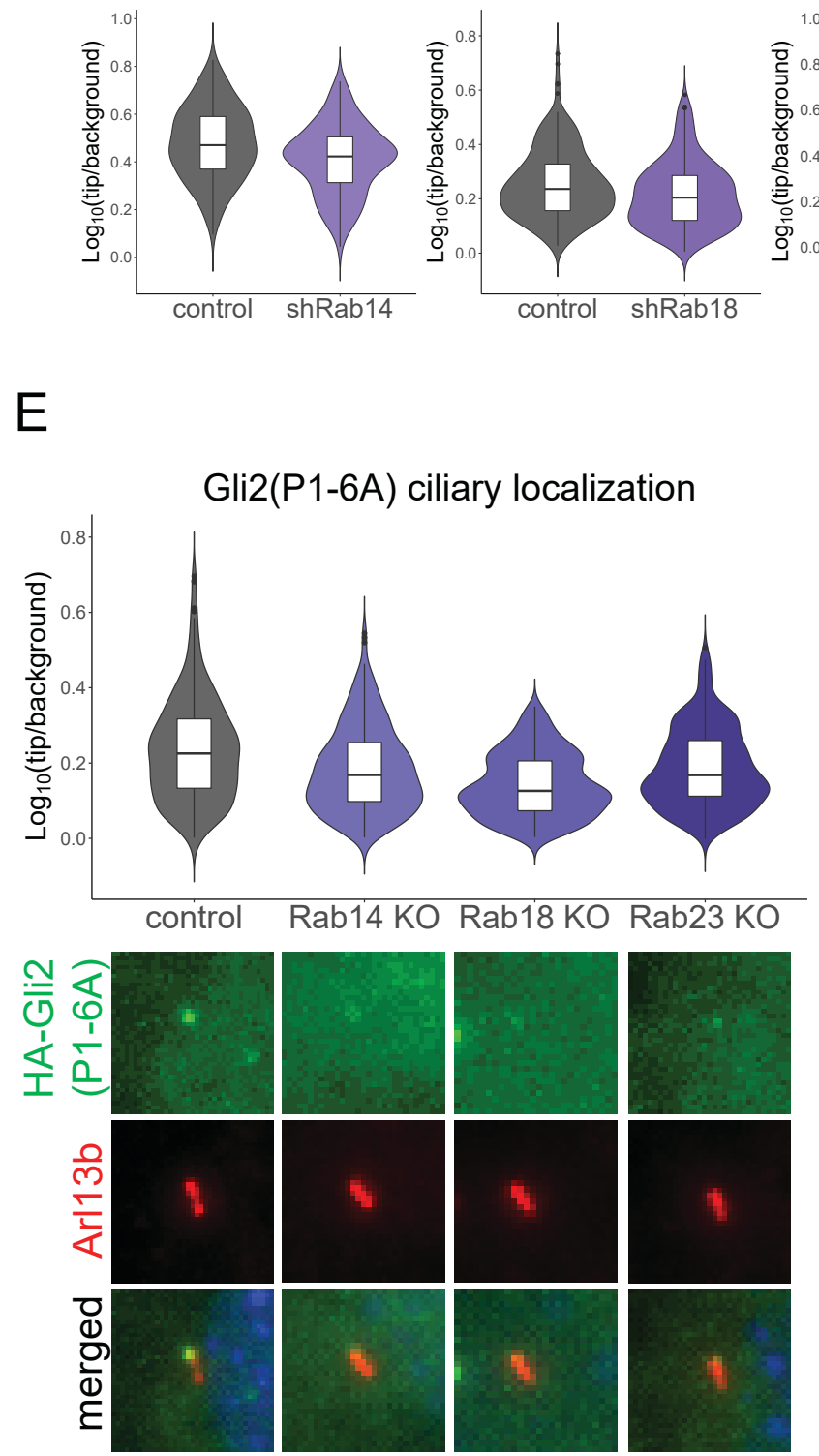

$\mathrm{F}$

B

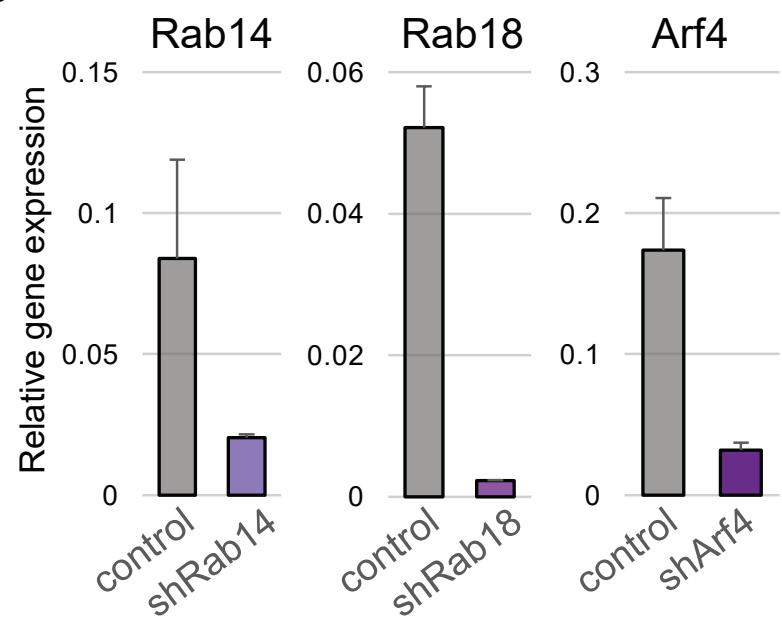

D

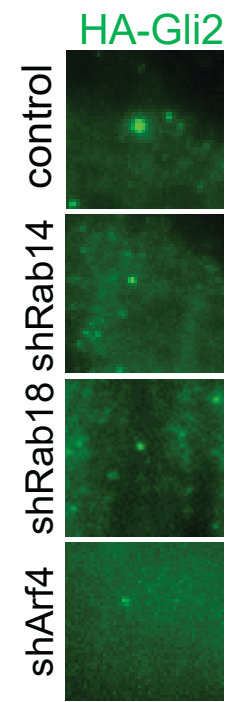

Gli2(P1-6A)

ciliary localization
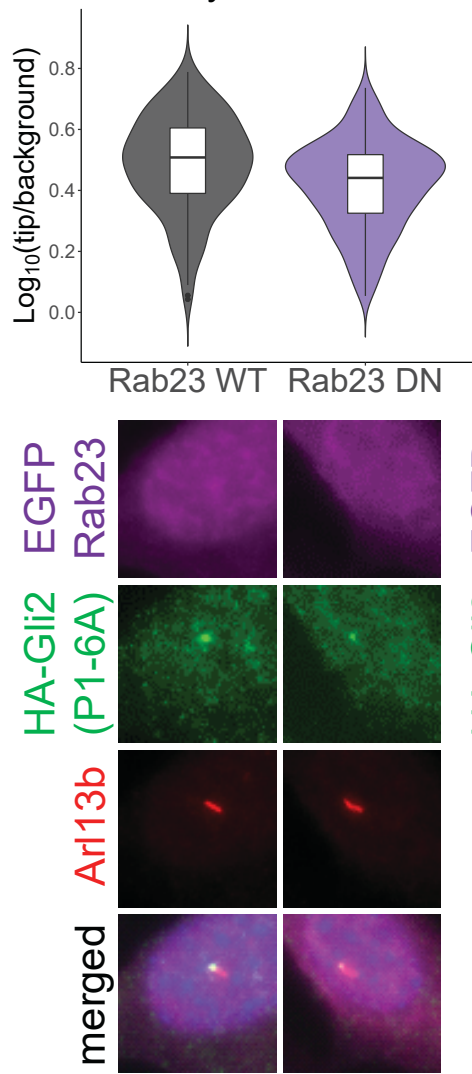

Gli2(P1-6A) ciliary localization
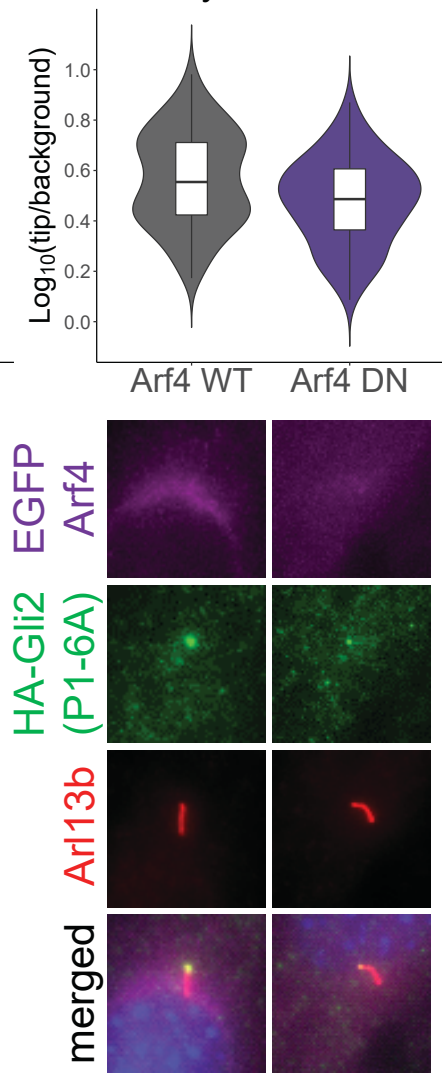
Fig. 7 Rab14, Rab18, Rab23, and Arf4 mediate Gli2 ciliary trafficking into primary cilium

(A) Co-immunoprecipitation of EGFP tagged Rab and Arf proteins with HA-Gli2(P1-6A). HEK293T cells were co-transfected with the indicated constructs and co-IP was performed as in Fig. 2C (B) Knockdown efficiency of Rab14, Rab18, and Arf4 using shRNA. Cells were transduced with viral constructs encoding the indicated shRNAs and mRNA expression of their target genes was measured by qRT-PCR. Control cells were transduced with the shRNA against luciferase (C) Effect of Rab14, Rab18, and Arf4 shRNA knockdown on relative Gli2(P1-6A) ciliary localization. Relative localization of Gli2(P1-6A) at the cilium tip was measured as in Fig. $3 \mathrm{C}$ for $\mathrm{n}>100$ cilia per group. Student's t-test control vs shRNA Rab14 $p$-value = 0.00018; control vs shRNA Rab18 $p$-value $=0.00027$; control vs shRNA Arf4 p-value $=0.0081$ (D) Representative images of HA-Gli2(P1-6A) localization in cilia of cells with the knockdown of Rab14, Rab18 and Arf4. Cells were transduced as in B. Arl13B was used as a ciliary marker (E) Effect of CRISPR-Cas9mediated knockout of Rab14, Rab18, and Rab23 on Gli2(P1-6A) ciliary localization. Cells stably expressing both HA-Gli2(P1-6A) and Cas9 were transduced with viral constructs encoding the indicated sgRNAs. Control cells were transduced with the empty pLentiGuide-puro vector. Relative localization of Gli2(P1-6A) at the cilium tip was measured as in Fig. $3 \mathrm{C}$ for $n>280$ cilia per group. Student's t-test control vs Rab14 edit $p$-value $=1.1 \mathrm{e}-06$; control vs Rab18 edit $\mathrm{p}$-value $<2.2 \mathrm{e}-16$; control vs Rab23 edit $\mathrm{p}$-value $=3.4 \mathrm{e}-07$. Representative images of Gli2(P1-6A) ciliary localization for each condition are presented below. Arl13b was used as a ciliary marker. (F) Effect of inducible expression of dominant-negative (DN) forms of Rab23 and Arf4 on Gli2(P1-6A) ciliary localization. Relative localization of Gli2(P1-6A) at the cilium tip was measured as in Fig. 3C for $n>100$ cilia per group. Student's t-test Rab23 WT vs DN p-value $=2.2 e-05$; Arf4 WT vs DN p-value $=0.00031$. Representative images of Gli2(P1-6A) ciliary localization for each condition are presented below. Arl13b was used as a ciliary marker. 
bioRxiv preprint doi: https://doi.org/10.1101/2021.09.27.461918; this version posted September 28, 2021. The copyright holder for this

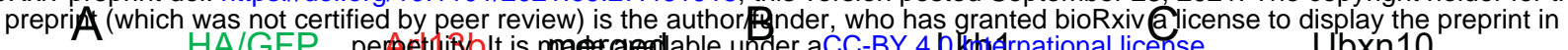
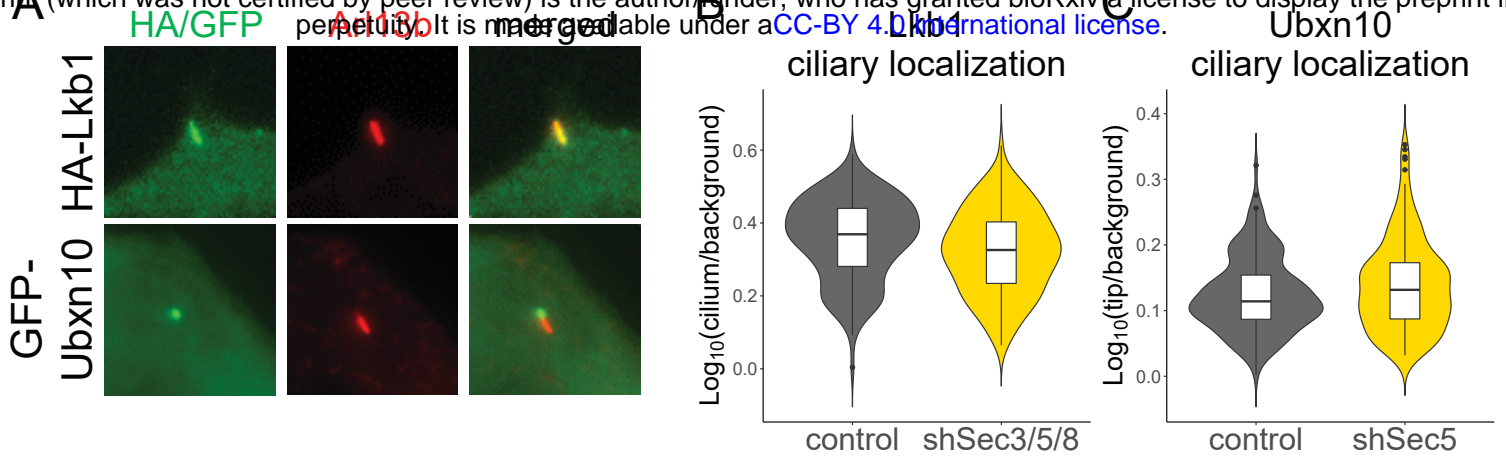

D

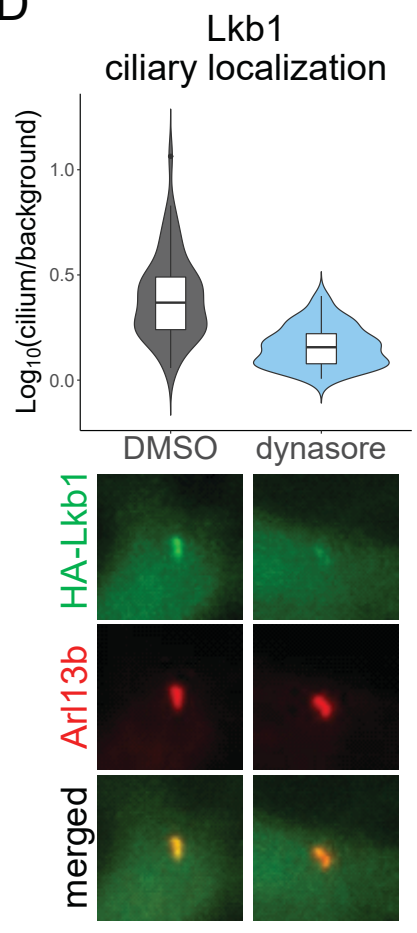

G

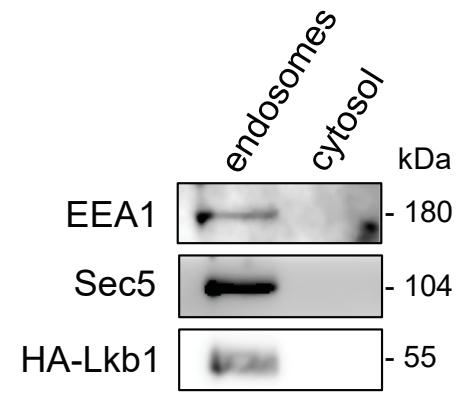

$E$

Ubxn10

ciliary localization

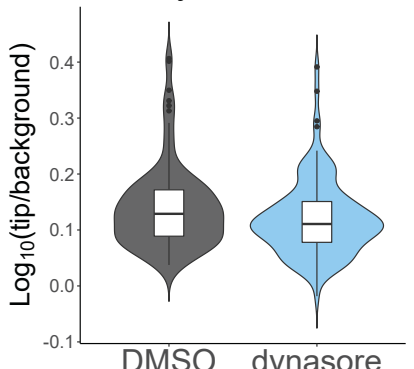

$\mathrm{F}$

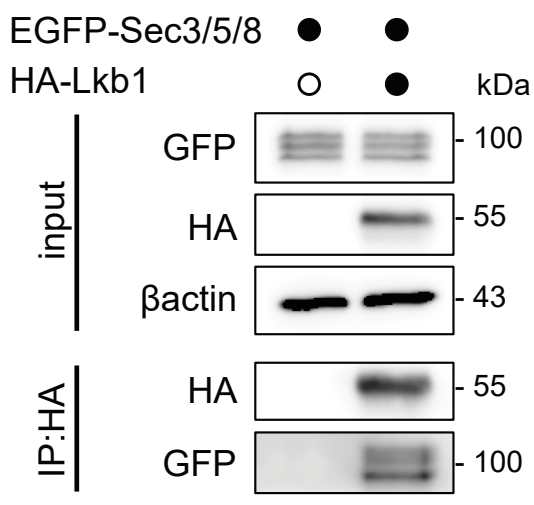

$\mathrm{H}$

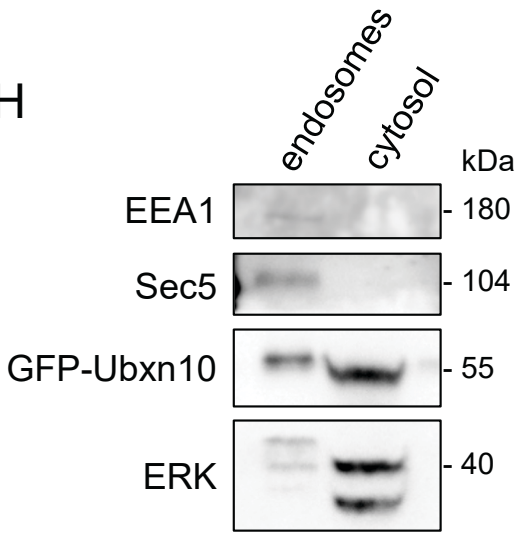

Fig. 8 The trafficking of Lkb1, but not Ubxn10, depends on endocytosis and the exocyst

(A) Ciliary localization of Lkb1 and Ubxn10 in NIH/3T3 cells. Cells were transfected and stained with the indicated antibodies. Arl13b was used as a ciliary marker. (B) Effect of Sec3/5/8 shRNA knockdown on Lkb1 ciliary localization. Cells were transduced as in Fig 3A. Relative localization of Lkb1 at the cilium tip was measured as in Fig. $3 \mathrm{C}$ for $n>$ 70 cilia per group. Student's t-test control vs shSec3/5/8 p-value $=p$-value $=0.003$ (C) Effect of Sec5 shRNA knockdown on relative Ubxn10 ciliary localization. Cells were transduced as in Fig $3 \mathrm{~A}$. Relative localization of Ubxn10 at the cilium tip was measured as in Fig. $3 C$ for $n>160$ cilia per group. Student's t-test control vs shSec5 $p$-value $=0.037$ (D) Effect of dynasore treatment on Lkb1 ciliary accumulation. NIH/3T3 cells with stable expression of HA-Lkb1 were treated with DMSO and dynasore $(4 \mathrm{~h} ; 40 \mu \mathrm{M})$. Relative localization of $L \mathrm{~kb} 1$ at the cilium was measured for $\mathrm{n}>50$ cells per group. Student's t-test p-value $=2.3 \mathrm{e}-11$. Representative images are presented below. $(E)$ Effect of dynasore treatment on Ubxn10 ciliary accumulation. Cells were treated as in B and relative localization was measured for $n>100$ cells per group. Student's t-test DMSO vs dynasore $4 \mathrm{~h} p$-value $=0.05$. Representative images are presented below. (F) Co-immunoprecipitation of EGFP tagged Sec3/5/8 proteins with HA-Lkb1 in HEK293T cells co-transfected with the indicated constructs $(\mathrm{G})$ Cells stably expressing HA-Lkb1 were fractionated using the endosome isolation kit and the fractions were resolved using SDS-PAGE. Immunoblot shows Lkb1 in the endosomal fraction. EEA1 was used as a marker of the endosomes. Silver-stained gel of the same samples shows similar total protein abundance in both fractions. $(\mathrm{H})$ Fractionation of stably expressed GFP-Ubxn10 cells as in G. Immunoblot shows Ubxn10 mainly in the cytosolic fraction. ERK was used as a cytosolic fraction marker. 


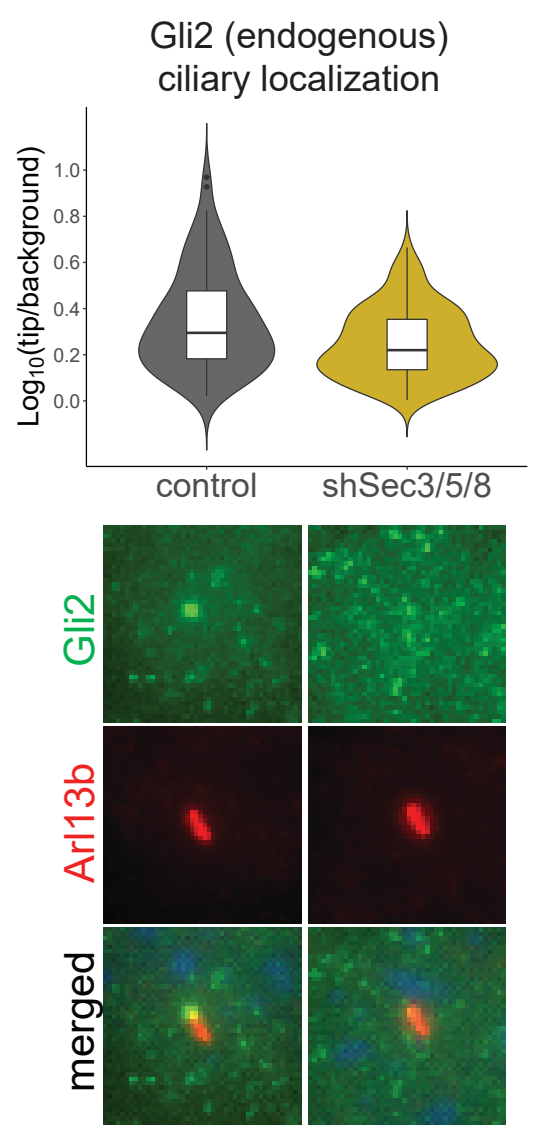

Figure S1. Relative localization at the cilium tip of endogenous Gli2 in NIH/3T3 cells with shRNA knockdown of Sec3, Sec5, and Sec8 and treated for 24h with SAG agonist. Results are presented as violin plots of log10-transformed ratios of fluorescence intensity of anti-HA staining at cilia tips to the intensity in the surrounding background. Cilia per variant $n>90$. Student's t-test analysis control-shSec $3 / 5 / 8$ $\mathrm{p}$-value $=0.0026$. Representative images of Gli2 ciliary localization for each condition are presented below. Arl13b was used as a ciliary marker. 
bioRxiv preprint doi: https://doi.org/10.1101/2021.09.27.461918; this version posted September 28, 2021. The copyright holder for this preprint (which was not certified by peer review) is the author/funder, who has granted bioRxiv a license to display the preprint in perpetuity. It is made available under aCC-BY 4.0 International license.

\section{GLI1}

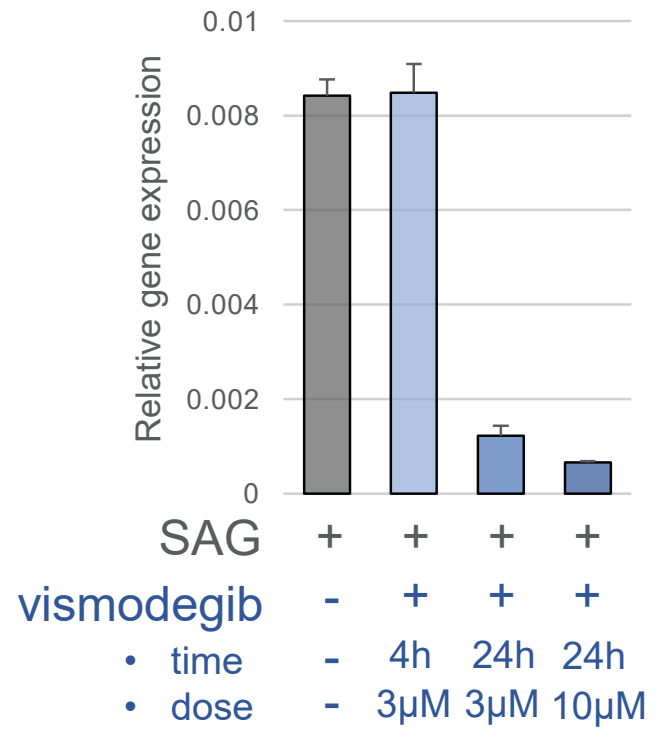

Figure S2 The relative mRNA expression level of Gli1 (Hh pathway activity marker) after indicated dose and time of vismogedib treatment. 
A

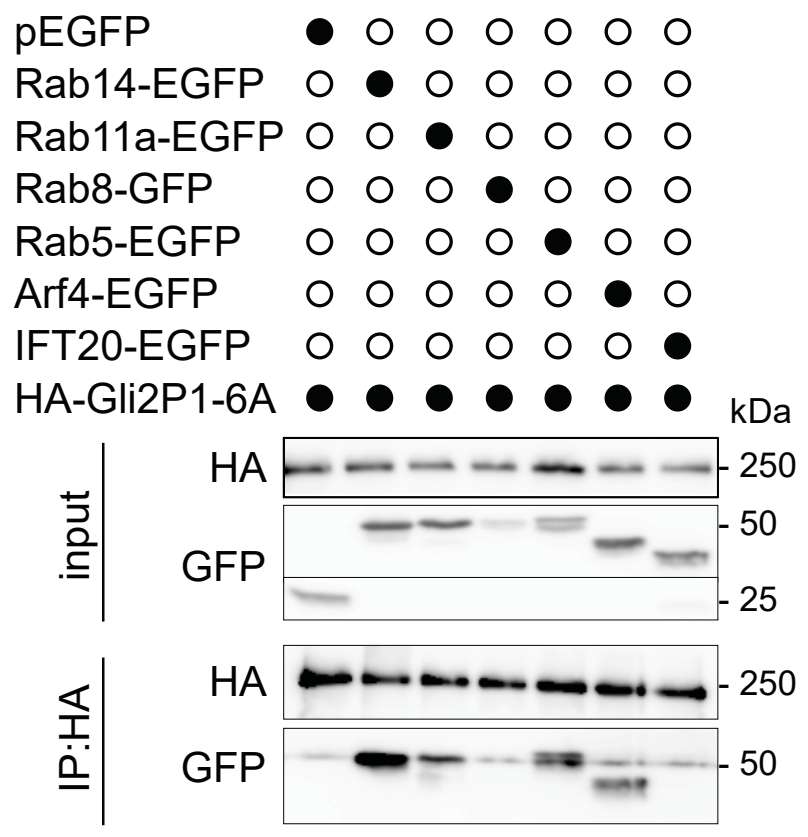

B

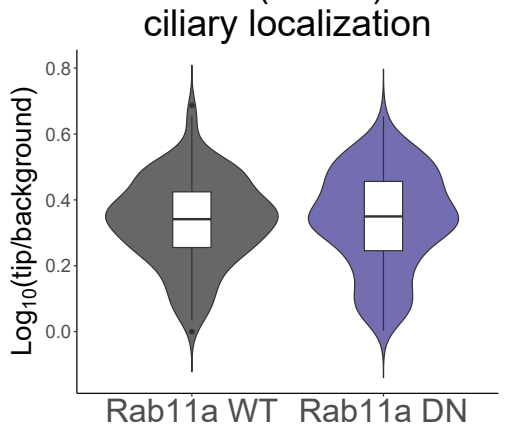

Gli2(P1-6A)

ciliary localization

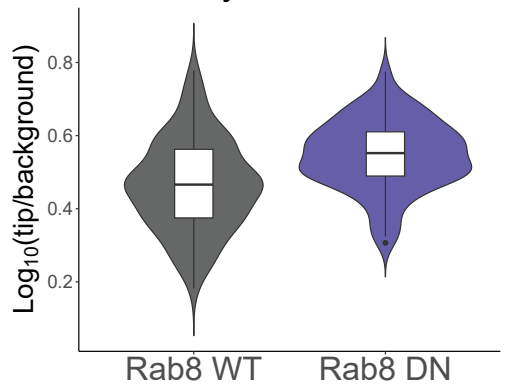

Figure S3 (A) Co-immunoprecipitation of EGFP tagged Rab, Arf, and IFT proteins with HA-Gli2(P16A). HEK293T cells were co-transfected with the indicated constructs and co-IP was performed using the HA beads. (B) Effect of inducible expression of dominant-negative (DN) forms of Rab8 and Rab11a on Gli2(P1-6A) ciliary localization. Relative localization of Gli2(P1-6A) at the cilium tip was measured as in Fig. 3C for $n>120$ cilia per group. Student's t-test Rab11a WT vs DN p-value $=0.70$; Rab8 WT vs DN p-value $=2.5 e-08$. 
bioRxiv preprint doi: https://doi.org/10.1101/2021.09.27.461918; this version posted September 28, 2021. The copyright holder for this preprint (which was not certified by peer review) is the author/funder, who has granted bioRxiv a license to display the preprint in perpetuity. It is made available under aCC-BY 4.0 International license.

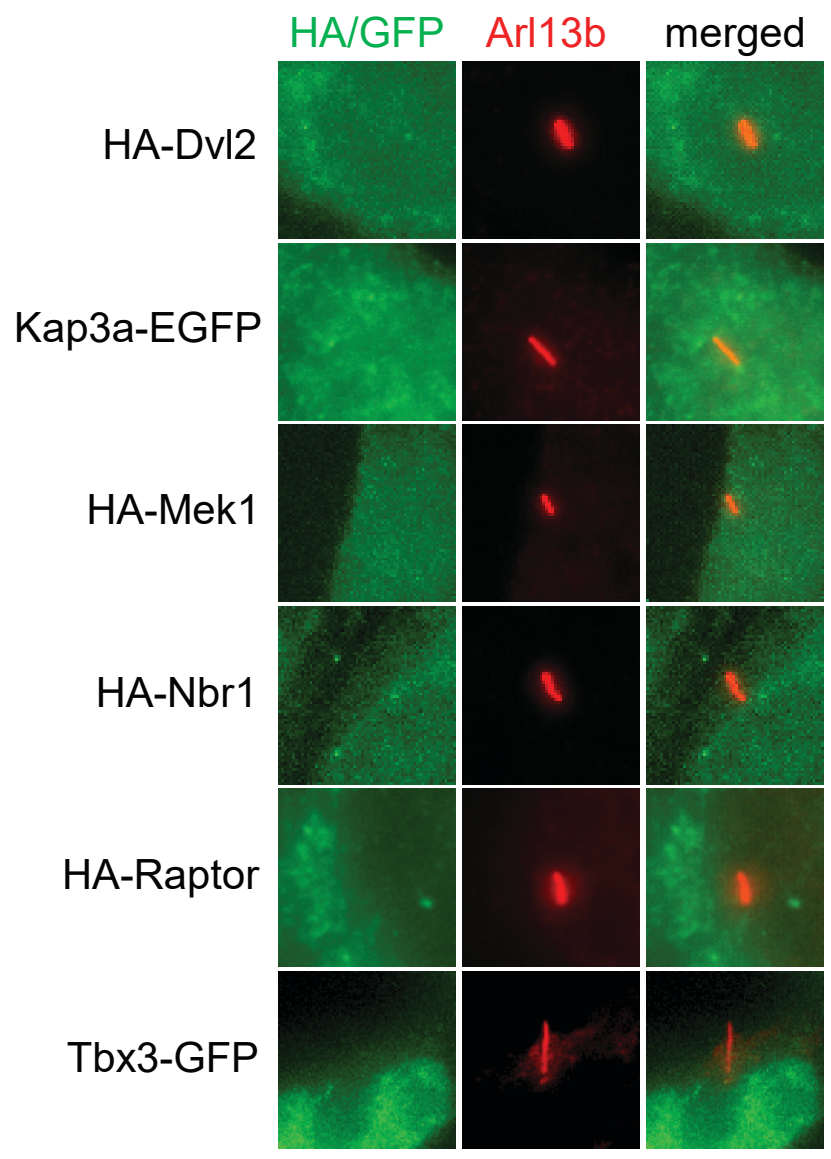

Figure S4 Ciliary localization of different putative ciliary proteins we tested in NIH/3T3 cells. Cells were transfected with indicated proteins tagged with HA or GFP and then we observed their ciliary localization. Arl13b was used as a ciliary marker. 